\title{
A Mixed Discontinuous Galerkin Approximation of Time Dependent Convection Diffusion Optimal Control Problem
}

\author{
Qingjin Xu and Zhaojie Zhou \\ College of Mathematical Sciences, Shandong Normal University, Jinan, China \\ Correspondence should be addressed to Zhaojie Zhou; zhouzhaojie@sdnu.edu.cn
}

Received 4 November 2016; Accepted 9 January 2017; Published 21 February 2017

Academic Editor: Ram Jiwari

Copyright (c) 2017 Qingjin Xu and Zhaojie Zhou. This is an open access article distributed under the Creative Commons Attribution License, which permits unrestricted use, distribution, and reproduction in any medium, provided the original work is properly cited.

\begin{abstract}
In this paper, we investigate a mixed discontinuous Galerkin approximation of time dependent convection diffusion optimal control problem with control constraints based on the combination of a mixed finite element method for the elliptic part and a discontinuous Galerkin method for the hyperbolic part of the state equation. The control variable is approximated by variational discretization approach. A priori error estimates of the state, adjoint state, and control are derived for both semidiscrete scheme and fully discrete scheme. Numerical example is given to show the effectiveness of the numerical scheme.
\end{abstract}

\section{Introduction}

The objective of this paper mainly focuses on developing a mixed discontinuous Galerkin scheme for the following control constrained optimal control problem governed by a transient convection diffusion equation:

$$
\begin{aligned}
\min _{u \in K} J(y, u) \\
:=\frac{1}{2} \int_{0}^{T} \int_{\Omega}\left(y(\mathbf{x}, t)-y_{d}(\mathbf{x}, t)\right)^{2} d x d t \\
\quad+\frac{\gamma}{2} \int_{0}^{T} \int_{\Omega} u^{2}(\mathbf{x}, t) d x d t
\end{aligned}
$$

subject to $\frac{\partial y}{\partial t}-\varepsilon \Delta y-\nabla \cdot(\beta y)+\alpha y=f+u$,

$$
\text { in } \Omega_{T}:=\Omega \times(0, T),
$$

$y=0, \quad$ on $\Gamma_{T}:=\partial \Omega \times(0, T)$,

$y(\mathbf{x}, 0)=y_{0}(\mathbf{x}), \quad$ in $\Omega$.

More details will be specified later.

This kind of problem plays an important role in many fields, such as air pollution ([1]) and waste water treatment ([2]). In recent years the research of numerical method for this kind of problem forms a hot topic. Lots of literatures are devoted to developing effective numerical methods for this kind of problem. In [3-6] the stabilization method such as local projection stabilization, SUPG, and continuous interior penalty method are investigated. The discontinuous Galerkin approximation including primary discontinuous Galerkin method and local discontinuous Galerkin method is addressed in [7-12]. In [13] the authors discuss the characteristic finite element approximation of transient convection diffusion optimal control problem. For more references, one can refer to [14].

In this paper, we investigate a mixed discontinuous Galerkin approximation of transient convection diffusion optimal control problem with control constraints. This scheme is based on the combination of a discontinuous Galerkin method for the hyperbolic part and a mixed finite element method for the elliptic part of the state equation. Variational discretization approach is utilized to approximate the control variable. The work of this paper is motivated by $[8,15]$ where a similar scheme was proposed for convection diffusion equations and stationary convection diffusion optimal control problems, respectively. Similar to other discontinuous Galerkin methods, this scheme is also locally conservative, which makes it much suitable for problems where conservation is important, for example, for time dependent convection diffusion problems. Moreover, when 
the diffusion coefficient tends to zero, this scheme reduces to the classical discontinuous Galerkin method. Thus it inherits the stabilizing features of discontinuous Galerkin methods. We derive a priori error estimates of the state, adjoint state, and control for both semidiscrete scheme and fully discrete scheme. Numerical experiment is carried out to show the performance of our scheme.

The rest of the paper is organized as follows: In Section 2, semidiscrete and fully discrete mixed discontinuous Galerkin scheme are defined for control constrained optimal control problems governed by the time dependent convection diffusion equations. In Section 3, a priori error estimates of the semidiscrete scheme are derived. In Section 4, we derive a priori error estimates of the fully discrete scheme. Finally numerical example is given to illustrate the theoretical findings.

\section{Mixed Discontinuous Galerkin Scheme}

Consider the following optimal control problem with control constraints:

$$
\begin{array}{cl}
\min _{u \in K} & J(y, u) \\
\text { subject to } & \frac{\partial y}{\partial t}-\varepsilon \Delta y-\nabla \cdot(\boldsymbol{\beta} y)+\alpha y=f+u, \\
& \text { in } \Omega_{T}:=\Omega \times(0, T), \\
& y=0, \quad \text { on } \Gamma_{T}:=\partial \Omega \times(0, T), \\
& y(\mathbf{x}, 0)=y_{0}(\mathbf{x}), \quad \text { in } \Omega,
\end{array}
$$

where $\Omega$ is a convex polygon with piecewise smooth boundary $\partial \Omega$. Here $K$ is the admissible set defined by

$$
\begin{aligned}
K & =\left\{q \in L^{2}\left(\Omega_{T}\right): a \leq q(x, t)\right. \\
& \left.\leq b \text { a.e. in } \Omega_{T} \text { with } a, b \in \mathrm{R}, a \leq b\right\} .
\end{aligned}
$$

$\alpha$ and $f$ are given functions, $\varepsilon>0$ is a constant, and $\beta$ is a given vector valued function, which satisfies $\alpha-(1 / 2) \nabla \cdot \beta \geq$ $\widetilde{\gamma}>0$ with a constant $\tilde{\gamma}>0$.

To define a mixed discontinuous Galerkin scheme for (2), we introduce a new variable:

$$
\mathbf{q}=-\varepsilon^{1 / 2} \nabla y
$$

Then the optimal control problem (2) can be rewritten as

$$
\begin{array}{rl}
\min _{u \in K} & J(y, u) \\
& y_{t}+\nabla \cdot\left(\varepsilon^{1 / 2} \mathbf{q}\right)-\nabla \cdot(\boldsymbol{\beta} y)+\alpha y=f+u, \\
& \quad \text { in } \Omega_{T}, \\
& \mathbf{q}=-\varepsilon^{1 / 2} \nabla y, \quad \text { in } \Omega_{T}, \\
y & =0, \quad \text { on } \Gamma_{T} .
\end{array}
$$

Let $T^{h}$ be a regular triangulation of $\Omega$ with element $\tau$ and $\bar{\Omega}=$ $\bigcup_{\tau \in T^{h}} \bar{\tau}$. Let $h=\max _{\tau \in T^{h}} h_{\tau}$, where $h_{\tau}$ denotes the diameter of the element $\tau$. We define the following spaces:

$$
\begin{aligned}
& \mathbf{V}=\left\{\mathbf{v} \in\left(L^{2}(\Omega)\right)^{2}: \nabla \cdot \mathbf{v} \in L^{2}(\Omega)\right\}, \\
& W=\left\{w \in L^{2}(\Omega):\left.w\right|_{\tau} \in H^{1}(\tau), \forall \tau \in T^{h}\right\}, \\
& U=L^{2}(\Omega) .
\end{aligned}
$$

For simplicity, we set

$$
\begin{aligned}
A(\mathbf{q}, \mathbf{v})= & (\mathbf{q}, \mathbf{v}), \quad \mathbf{q}, \mathbf{v} \in \mathbf{V}, \\
B(\mathbf{q}, w)= & \left(\operatorname{div}\left(\varepsilon^{1 / 2} \mathbf{q}\right), w\right), \quad \mathbf{q} \in \mathbf{V}, w \in W, \\
D(y, w)= & \sum_{\tau \in T^{h}}\left(\int_{\tau} y \boldsymbol{\beta} \cdot \nabla w-\int_{\partial \tau} y_{+}[w] \mathbf{n} \cdot \boldsymbol{\beta} d s\right) \\
& +(\alpha y, w), \quad y, w \in W,
\end{aligned}
$$

where

$$
\partial \tau_{-}=\left\{l \in \partial \tau,\left.\mathbf{n} \cdot \boldsymbol{\beta}\right|_{l}<0\right\},
$$

$\mathbf{n}$ is the outward norm direction on $\partial \tau$,

$$
\begin{aligned}
{[w] } & =w_{+}-w_{-}, \\
w_{+}(x) & =\lim _{t \rightarrow 0^{+}} w(x+t \boldsymbol{\beta}), \\
w_{-}(x) & =\lim _{t \rightarrow 0^{-}} w(x+t \boldsymbol{\beta}),
\end{aligned}
$$

and $[w]=w_{+}$on $\partial \tau$ when $\partial \tau_{-} \subset \partial \Omega$. Then the weak formulation for the optimal control problem (5) reads as follows: finding $(\mathbf{q}, y, u) \subset \mathbf{V} \times W \times K$ such that

$$
\min _{u \in K} J(y, u)
$$

subject to $\left(y_{t}, w\right)+B(\mathbf{q}, w)+D(y, w)$

$$
\begin{aligned}
& =(f+u, w), \quad \forall w \in W \\
& A(\mathbf{q}, \mathbf{v})-B(\mathbf{v}, y)=0, \quad \forall \mathbf{v} \in \mathbf{V}, \\
& y(\mathbf{x}, 0)=y_{0}(\mathbf{x}), \quad \forall \mathbf{x} \in \Omega .
\end{aligned}
$$

Standard arguments techniques imply that optimal control problem (10)-(11) admits a unique solution and the following first-order optimality condition holds:

$$
\begin{array}{r}
\left(y_{t}, w\right)+B(\mathbf{q}, w)+D(y, w)=(f+u, w), \\
A(\mathbf{q}, \mathbf{v})-B(\mathbf{v}, y)=0, \quad \forall \mathbf{v} \in \mathbf{V}, \\
-\left(z_{t}, \psi\right)-B(\mathbf{p}, \psi)+D(\psi, z)=\left(y-y_{d}, \psi\right),
\end{array}
$$

$\forall w \in W$

$\forall \psi \in W$

$$
\begin{gathered}
A(\mathbf{p}, \boldsymbol{\varphi})+B(\boldsymbol{\varphi}, z)=0, \quad \forall \boldsymbol{\varphi} \in \mathbf{V}, \\
\int_{0}^{T}(\gamma u+z, \widetilde{v}-u) d t \geq 0, \quad \forall \widetilde{v} \in K,
\end{gathered}
$$




$$
\begin{aligned}
& y(\mathbf{x}, 0)=y_{0}(\mathbf{x}), \\
& z(\mathbf{x}, T)=0
\end{aligned}
$$

$\forall \mathbf{x} \in \Omega$.

Let $\mathbf{V}_{h} \times W_{h} \subset \mathbf{V} \times W$ denote the Raviart-Thomas element space of the lowest order associated with a triangular or rectangular mesh $T^{h}$ of $\Omega$ (see [16] for details). Then the approximation scheme of (10)-(11) is as follows: finding $\left(\mathbf{q}_{h}, y_{h}, u_{h}\right) \in \mathbf{V}_{h} \times W_{h} \times K$ such that

$$
\min _{u_{h} \in K} J(y, u)
$$

subject to $\left(y_{h, t}, w_{h}\right)+B\left(\mathbf{q}_{h}, w_{h}\right)+D\left(y_{h}, w_{h}\right)$

$$
\begin{aligned}
& =\left(f+u_{h}, w_{h}\right), \quad \forall w_{h} \in W_{h}, \\
& A\left(\mathbf{q}_{h}, \mathbf{v}_{h}\right)-B\left(\mathbf{v}_{h}, y_{h}\right)=0, \quad \forall \mathbf{v}_{h} \in \mathbf{V}_{h}, \\
& y_{h}(\mathbf{x}, 0)=\tilde{y}_{0}, \quad \forall \mathbf{x} \in \Omega .
\end{aligned}
$$

Here $\tilde{y}_{0}$ is an approximation of the initial value $y_{0}(\mathbf{x})$. Variational discretization approach is used for the control $u$.

Similar to continuous case we derive the following semidiscrete optimality conditions:

$$
\begin{aligned}
\left(y_{h, t}, w_{h}\right)+B\left(\mathbf{q}_{h}, w_{h}\right)+D\left(y_{h}, w_{h}\right)=\left(f+u_{h}, w_{h}\right), & \forall w_{h} \in W_{h}, \\
A\left(\mathbf{q}_{h}, \mathbf{v}_{h}\right)-B\left(\mathbf{v}_{h}, y_{h}\right)=0, & \forall \mathbf{v}_{h} \in \mathbf{V}_{h}, \\
-\left(z_{h, t}, \psi_{h}\right)-B\left(\mathbf{p}_{h}, \psi_{h}\right)+D\left(\psi_{h}, z_{h}\right)=\left(y_{h}-y_{d}, \psi_{h}\right), & \forall \psi_{h} \in W_{h}, \\
A\left(\mathbf{p}_{h}, \boldsymbol{\varphi}_{h}\right)+B\left(\boldsymbol{\varphi}_{h}, z_{h}\right)=0, & \\
\int_{0}^{T}\left(\gamma u_{h}+z_{h}, \widetilde{v}_{h}-u_{h}\right) d t \geq 0, & \forall \widetilde{v}_{h} \in K, \\
y_{h}(\mathbf{x}, 0)=\tilde{y}_{0}, & \\
z_{h}(\mathbf{x}, T)=0, &
\end{aligned}
$$

$\forall \mathbf{x} \in \Omega$.

To define a fully discrete scheme we introduce a time partition. Let $0=t_{0}<t_{1}<\cdots<t_{N-1}<t_{N}=T$ be a time grid with $k=t_{n}-t_{n-1}, n=1,2, \ldots, N$. Let $I_{n}=\left(t_{n-1}, t_{n}\right]$ be a half-open interval. We write $\phi_{n}:=\phi\left(t_{n}\right)$ for a smooth $\phi$.

We use backward Euler scheme for time discretization. Then a fully discrete scheme of (10)-(11) is characterized as follows: finding $\left(\mathbf{q}_{h}^{n}, y_{h}^{n}, u_{h}^{n}\right) \in \mathbf{V}_{h} \times W_{h} \times K$ such that

$$
\begin{aligned}
\min _{u_{h}^{n} \in K} & J_{h, k}\left(y_{h}^{n}, u_{h}^{n}\right) \\
& :=\frac{1}{2} \sum_{n=1}^{N} k\left(\left\|y_{h}^{n}-y_{d}^{n}\right\|_{0, \Omega}^{2}+\gamma\left\|u_{h}^{n}\right\|_{0, \Omega}^{2}\right)
\end{aligned}
$$

$$
\begin{aligned}
& \text { subject to }\left(\frac{y_{h}^{n}-y_{h}^{n-1}}{k}, w_{h}\right)+B\left(\mathbf{q}_{h}^{n}, w_{h}\right) \\
& +D\left(y_{h}^{n}, w_{h}\right)=\left(f^{n}+u_{h}^{n}, w_{h}\right),
\end{aligned}
$$

$$
\begin{array}{ll}
A\left(\mathbf{q}_{h}^{n}, \mathbf{v}_{h}\right)-B\left(\mathbf{v}_{h}, y_{h}^{n}\right)=0, \quad \forall w_{h} \in W_{h}, \\
y_{h}^{0}=\tilde{y}_{0}, \quad \forall \mathbf{x} \in \Omega . \mathbf{V}_{h},
\end{array}
$$

To obtain the fully discrete first-order optimality condition we define a Lagrange functional as follows:

$$
\begin{aligned}
& L\left(\mathscr{Y}_{h}, \mathscr{Q}_{h}, \mathscr{Z}_{h}, \mathscr{P}_{h}, \mathscr{U}_{h}\right) \\
& \quad:=k \sum_{n=1}^{N}\left[\left(f^{n}+u_{h}^{n}-\frac{y_{h}^{n}-y_{h}^{n-1}}{k}, z_{h}^{n-1}\right)\right. \\
& \quad-B\left(\mathbf{q}_{h}^{n}, z_{h}^{n-1}\right)-D\left(y_{h}^{n}, z_{h}^{n-1}\right)+B\left(\mathbf{p}_{h}^{n-1}, y_{h}^{n}\right) \\
& \left.\quad-A\left(\mathbf{q}_{h}^{n}, \mathbf{p}_{h}^{n-1}\right)\right]+J_{h, k}\left(y_{h}^{n}, u_{h}^{n}\right) .
\end{aligned}
$$

Here $\mathscr{Y}_{h}=\left(y_{h}^{1}, y_{h}^{2}, \ldots, y_{h}^{N}\right)^{T}, Q_{h}=\left(\mathbf{q}_{h}^{1}, \mathbf{q}_{h}^{2}, \ldots, \mathbf{q}_{h}^{N}\right)^{T}$, $\mathscr{Z}_{h}=\left(z_{h}^{0}, z_{h}^{1}, \ldots, z_{h}^{N-1}\right)^{T}, \mathscr{P}_{h}=\left(\mathbf{p}_{h}^{0}, \mathbf{p}_{h}^{1}, \ldots, \mathbf{p}_{h}^{N-1}\right)^{T}$, and $\mathscr{U}_{h}=\left(u_{h}^{1}, u_{h}^{2}, \ldots, u_{h}^{N}\right)^{T}$. Then we derive the discrete firstorder optimality condition:

$$
\begin{aligned}
& \left(\frac{y_{h}^{n}-y_{h}^{n-1}}{k}, w_{h}\right)+B\left(\mathbf{q}_{h}^{n}, w_{h}\right)+D\left(y_{h}^{n}, w_{h}\right) \\
& =\left(f^{n}+u_{h}^{n}, w_{h}\right), \quad \forall w_{h} \in W_{h}, \\
& A\left(\mathbf{q}_{h}^{n}, \mathbf{v}_{h}\right)-B\left(\mathbf{v}_{h}, y_{h}^{n}\right)=0, \quad \forall \mathbf{v}_{h} \in \mathbf{V}_{h}, \\
& \left(\frac{z_{h}^{n-1}-z_{h}^{n}}{k}, \psi_{h}\right)-B\left(\mathbf{p}_{h}^{n-1}, \psi_{h}\right)+D\left(\psi_{h}, z_{h}^{n-1}\right) \\
& =\left(y_{h}^{n}-y_{d}^{n}, \psi_{h}\right), \quad \forall \psi_{h} \in W_{h}, \\
& A\left(\mathbf{p}_{h}^{n-1}, \boldsymbol{\varphi}_{h}\right)+B\left(\boldsymbol{\varphi}_{h}, z_{h}^{n-1}\right)=0, \quad \forall \boldsymbol{\varphi}_{h} \in \mathbf{V}_{h}, \\
& \left(\gamma u_{h}^{n}+z_{h}^{n-1}, \widetilde{v}-u_{h}^{n}\right) \geq 0, \quad \forall \widetilde{v} \in K,
\end{aligned}
$$

$$
y_{h}^{0}=\tilde{y}_{0}
$$$$
z_{h}^{N}=0
$$

$\forall \mathbf{x} \in \Omega$.

\section{Semidiscrete Error Estimate}

The goal of this section is to prove the semidiscrete error estimates for the state, the adjoint state, and the control. We firstly decompose $y-y_{h}$ and $z-z_{h}$ as

$$
\begin{aligned}
& y-y_{h}=y(u)-y_{h}(u)+y_{h}(u)-y_{h}, \\
& z-z_{h}=z(u)-z_{h}(u)+z_{h}(u)-z_{h},
\end{aligned}
$$


where $y_{h}(u)$ and $z_{h}(u)$ satisfy the following auxiliary problems:

$$
\begin{aligned}
& \left(y_{h, t}(u), w_{h}\right)+B\left(\mathbf{q}_{h}(u), w_{h}\right)+D\left(y_{h}(u), w_{h}\right) \\
& \quad=\left(f+u, w_{h}\right), \\
& A\left(\mathbf{q}_{h}(u), \mathbf{v}_{h}\right)-B\left(\mathbf{v}_{h}, y_{h}(u)\right)=0, \\
& -\left(z_{h, t}(u), \psi_{h}\right)-B\left(\mathbf{p}_{h}(u), \psi_{h}\right)+D\left(\psi_{h}, z_{h}(u)\right) \\
& \quad=\left(y_{h}(u)-y_{d}, \psi_{h}\right), \\
& A\left(\mathbf{p}_{h}(u), \boldsymbol{\varphi}_{h}\right)+B\left(\boldsymbol{\varphi}_{h}, z_{h}(u)\right)=0, \\
& y_{h}(u)(\mathbf{x}, 0)=\tilde{y}_{0}, \\
& z_{h}(u)(\mathbf{x}, T)=0 .
\end{aligned}
$$

For the following analysis we introduce a new norm:

$$
\begin{aligned}
\|y\|_{*}^{2}= & \tilde{\gamma}\|y\|_{0, \Omega}^{2}+\frac{1}{2} \sum_{\tau \in T^{h}} \int_{\partial \tau_{-}}[y]^{2}|\mathbf{n} \cdot \boldsymbol{\beta}| d s \\
& +\frac{1}{2} \int_{\partial \Omega \mid\left(\bigcup \partial \tau_{-}\right)} y_{-}^{2}|\mathbf{n} \cdot \boldsymbol{\beta}| d s .
\end{aligned}
$$

Following [8] we have $D(y, y) \geq\|y\|_{*}^{2}$.

Let $(\widetilde{y}, \widetilde{\mathbf{q}}) \in W_{h} \times \mathbf{V}_{h}$ and $(\widetilde{z}, \widetilde{\mathbf{p}}) \in W_{h} \times \mathbf{V}_{h}$ be the projections defined by

$$
\begin{aligned}
B\left(\mathbf{q}-\widetilde{\mathbf{q}}, w_{h}\right)+D\left(y-\tilde{y}, w_{h}\right)=0, & \forall w_{h} \in W_{h}, \\
A\left(\mathbf{q}-\widetilde{\mathbf{q}}, \mathbf{v}_{h}\right)-B\left(\mathbf{v}_{h}, y-\tilde{y}\right)=0, & \forall \mathbf{v}_{h} \in \mathbf{V}_{h}, \\
D\left(\psi_{h}, z-\widetilde{z}\right)-B\left(\mathbf{p}-\widetilde{\mathbf{p}}, \psi_{h}\right)=0, & \forall \psi_{h} \in W_{h}, \\
A\left(\mathbf{p}-\widetilde{\mathbf{p}}, \boldsymbol{\varphi}_{h}\right)+B\left(\boldsymbol{\varphi}_{h}, z-\widetilde{z}\right)=0, & \forall \boldsymbol{\varphi}_{h} \in \mathbf{V}_{h},
\end{aligned}
$$

According to [8] we have the following.

Lemma 1. If $(\widetilde{y}, \widetilde{\mathbf{q}})$ and $(\widetilde{z}, \widetilde{\mathbf{p}})$ satisfy (21)-(22), respectively, then we have

$$
\begin{gathered}
\|\mathbf{q}-\widetilde{\mathbf{q}}\|_{0, \Omega}^{2}+\|y-\tilde{y}\|_{*}^{2} \leq \operatorname{Ch}\left(\|\mathbf{q}\|_{1 / 2, \Omega}^{2}+\|y\|_{1, \Omega}^{2}\right), \\
\|\mathbf{p}-\widetilde{\mathbf{p}}\|_{0, \Omega}^{2}+\|z-\widetilde{z}\|_{*}^{2} \leq \operatorname{Ch}\left(\|\mathbf{p}\|_{1 / 2, \Omega}^{2}+\|z\|_{1, \Omega}^{2}\right) .
\end{gathered}
$$

Lemma 2. If $(\widetilde{y}, \widetilde{\mathbf{q}})$ and $(\widetilde{z}, \widetilde{\mathbf{p}})$ satisfy (21)-(22), respectively, then the following estimates hold:

$$
\begin{gathered}
\left\|\mathbf{q}_{t}-\widetilde{\mathbf{q}}_{t}\right\|_{0, \Omega}^{2}+\left\|y_{t}-\tilde{y}_{t}\right\|_{*}^{2} \leq \operatorname{Ch}\left(\left\|\mathbf{q}_{t}\right\|_{1 / 2, \Omega}^{2}+\left\|y_{t}\right\|_{1, \Omega}^{2}\right), \\
\left\|\mathbf{p}_{t}-\widetilde{\mathbf{p}}_{t}\right\|_{0, \Omega}^{2}+\left\|z_{t}-\widetilde{z}_{t}\right\|_{*}^{2} \leq \operatorname{Ch}\left(\|\mathbf{p}\|_{1 / 2, \Omega}^{2}+\|z\|_{1, \Omega}^{2}\right) .
\end{gathered}
$$

Proof. Differentiating (21) about $t$, we have

$$
\begin{gathered}
B\left(\mathbf{q}_{t}-\widetilde{\mathbf{q}}_{t}, w_{h}\right)+D\left(y_{t}-\tilde{y}_{t}, w_{h}\right)=0, \quad \forall w_{h} \in W_{h}, \\
A\left(\mathbf{q}_{t}-\widetilde{\mathbf{q}}_{t}, \mathbf{v}_{h}\right)-B\left(\mathbf{v}_{h}, y_{t}-\tilde{y}_{t}\right)=0, \quad \forall \mathbf{v}_{h} \in \mathbf{V}_{h} .
\end{gathered}
$$

Set $P_{h}: W \rightarrow W_{h}$ and $\Pi_{h}: \mathbf{V} \rightarrow \mathbf{V}_{h}$ to be the interpolation operators for the standard RT element space (see, e.g., [17]) such that

$$
\begin{aligned}
\left(\psi-P_{h} \psi, w_{h}\right)=0, & \forall w_{h} \in W_{h}, \\
\left(\operatorname{div}\left(\phi-\Pi_{h} \phi\right), w_{h}\right)=0, & \forall w_{h} \in W_{h} .
\end{aligned}
$$

Then we have the following approximation properties:

$$
\begin{gathered}
\left\|\psi-P_{h} \psi\right\|_{s, \Omega} \leq C h^{1-s}\|\psi\|_{1, \Omega}, \\
s=0,1, \quad \forall \psi \in H_{0}^{1}(\Omega), \\
\left\|\phi-\Pi_{h} \phi\right\|_{0, \Omega} \leq C h^{\gamma}|\phi|_{\gamma, \Omega}, \\
\frac{1}{2} \leq \gamma<1, \quad \forall \phi \in\left(H_{0}^{\gamma}(\Omega)\right)^{2} .
\end{gathered}
$$

Let

$$
\begin{aligned}
& \mathbf{q}-\widetilde{\mathbf{q}}=\mathbf{q}-\Pi_{h} \mathbf{q}+\Pi_{h} \mathbf{q}-\widetilde{\mathbf{q}}=\eta_{\mathbf{q}}+\xi_{\mathbf{q}}, \\
& y-\tilde{y}=y-P_{h} y+P_{h} y-\tilde{y}=\eta_{y}+\xi_{y} .
\end{aligned}
$$

Then we derive

$$
\begin{gathered}
B\left(\eta_{\mathbf{q}, t}, w_{h}\right)+B\left(\xi_{\mathbf{q}, t}, w_{h}\right)+D\left(\eta_{y, t}, w_{h}\right) \\
+D\left(\xi_{y, t}, w_{h}\right)=0, \quad \forall w_{h} \in W_{h}, \\
A\left(\eta_{\mathbf{q}, t}, \mathbf{v}_{h}\right)+A\left(\xi_{\mathbf{q}, t}, \mathbf{v}_{h}\right)-B\left(\mathbf{v}_{h}, \eta_{y, t}\right) \\
-B\left(\mathbf{v}_{h}, \xi_{y, t}\right)=0, \quad \forall \mathbf{v}_{h} \in \mathbf{V}_{h} .
\end{gathered}
$$

By the definition of interpolation operators $P_{h}$ and $\Pi_{h}$ we obtain

$$
\begin{gathered}
B\left(\eta_{\mathbf{q}, t}, w_{h}\right)=0, \quad \forall w_{h} \in W_{h}, \\
B\left(\mathbf{v}_{h}, \eta_{y, t}\right)=0, \quad \forall \mathbf{v}_{h} \in \mathbf{V}_{h} .
\end{gathered}
$$

Setting $w_{h}=\xi_{y, t}$ and $\mathbf{v}_{h}=\xi_{\mathbf{q}, t}$ leads to

$$
\begin{aligned}
& A\left(\xi_{\mathbf{q}, t}, \xi_{\mathbf{q}, t}\right)+D\left(\xi_{y, t}, \xi_{y, t}\right) \\
& =A\left(\eta_{\mathbf{q}, t},-\xi_{\mathbf{q}, t}\right)+D\left(\eta_{y, t},-\xi_{y, t}\right),
\end{aligned}
$$

namely,

$$
\left\|\xi_{\mathbf{q}, t}\right\|_{0, \Omega}^{2}+\left\|\xi_{y, t}\right\|_{*}^{2} \leq A\left(\eta_{\mathbf{q}, t},-\xi_{\mathbf{q}, t}\right)+D\left(\eta_{y, t},-\xi_{y, t}\right) .
$$

Since the function in $W_{h}$ is piecewise constant, using the definition of $D(\cdot, \cdot)$, we have for all $w \in W$ and $\theta \in W_{h}$

$$
\begin{aligned}
D(w, \theta)= & \sum_{\tau \in T^{h}}\left(\int_{\tau} w \beta \cdot \nabla \theta-\int_{\partial} \tau_{-} w_{+}[\theta] \mathbf{n} \cdot \boldsymbol{\beta} d s\right) \\
& +(\alpha w, \theta) \\
= & 0-\sum_{\tau \in T^{h}} \int_{\partial \tau_{-}} w_{+}[\theta] \mathbf{n} \cdot \boldsymbol{\beta} d s+(\alpha w, \theta) .
\end{aligned}
$$


Therefore, we get the estimate as follows:

$$
\begin{aligned}
& \left\|\xi_{\mathbf{q}, t}\right\|_{0, \Omega}^{2}+\left\|\xi_{y, t}\right\|_{*}^{2} \leq C(\delta)\left(\left\|\eta_{\mathbf{q}, t}\right\|_{0, \Omega}^{2}+\left\|\eta_{y, t}\right\|_{0, \Omega}^{2}\right) \\
& +C(\delta) \sum_{\tau} \int_{\partial \tau_{-}}\left(\eta_{y, t}\right)_{+}^{2}|\mathbf{n} \cdot \boldsymbol{\beta}| d s+C \delta\left(\left\|\xi_{\mathbf{q}, t}\right\|_{0, \Omega}^{2}\right. \\
& \left.+\left\|\xi_{y, t}\right\|_{0, \Omega}^{2}+\sum_{\tau} \int_{\partial \tau_{-}}\left[\xi_{y, t}\right]^{2}|\mathbf{n} \cdot \boldsymbol{\beta}| d s\right)
\end{aligned}
$$

Note that

$$
\begin{aligned}
& \left\|\mathbf{q}-\Pi_{h} \mathbf{q}\right\|_{0, \Omega} \leq C h^{1 / 2}\|\mathbf{q}\|_{1 / 2, \Omega}, \\
& \left\|y-P_{h} y\right\|_{0, \Omega} \leq C h\|y\|_{1, \Omega},
\end{aligned}
$$

and according to [18] we have

$$
\begin{gathered}
\sum_{\tau \in T^{h}} \int_{\partial \tau_{-}}\left(\eta_{y, t}\right)_{+}^{2}|\mathbf{n} \cdot \boldsymbol{\beta}| d s \leq C \sum_{\tau \in T^{h}}\left\|\eta_{y, t}\right\|_{0, \partial \tau}^{2} \\
\leq C \sum_{\tau \in T^{h}}\left\|\eta_{y, t}\right\|_{0, \tau}\left\|\eta_{y, t}\right\|_{1, \tau} \leq C h\left\|y_{t}\right\|_{1, \Omega}^{2} .
\end{gathered}
$$

Then we derive

$$
\begin{aligned}
\left\|\xi_{\mathbf{q}, t}\right\|_{0, \Omega}^{2}+\left\|\xi_{y, t}\right\|_{*}^{2} \leq & C(\delta) h\left(\left\|\mathbf{q}_{t}\right\|_{1 / 2, \Omega}^{2}+\left\|y_{t}\right\|_{1, \Omega}^{2}\right) \\
& +C \delta\left(\left\|\xi_{\mathbf{q}, t}\right\|_{0, \Omega}^{2}+\left\|\xi_{y, t}\right\|_{*}^{2}\right) .
\end{aligned}
$$

By setting $\delta$ small enough we obtain

$$
\left(\left\|\xi_{\mathbf{q}, t}\right\|_{0, \Omega}^{2}+\left\|\xi_{y, t}\right\|_{*}^{2}\right)^{1 / 2} \leq C h^{1 / 2}\left(\left\|\mathbf{q}_{t}\right\|_{1 / 2, \Omega}+\left\|y_{t}\right\|_{1, \Omega}\right) .
$$

Using triangle inequality we have

$$
\begin{aligned}
& \left\|\mathbf{q}_{t}-\tilde{\mathbf{q}}_{t}\right\|_{0, \Omega}^{2}+\left\|y_{t}-\tilde{y}_{t}\right\|_{*}^{2} \\
& \quad \leq\left\|\eta_{\mathbf{q}, t}\right\|_{0, \Omega}^{2}+\left\|\eta_{y, t}\right\|_{*}^{2}+\left\|\xi_{\mathbf{q}, t}\right\|_{0, \Omega}^{2}+\left\|\xi_{y, t}\right\|_{*}^{2},
\end{aligned}
$$

which implies the first theorem result. Similar to the above proof we have

$$
\left\|\mathbf{p}_{t}-\widetilde{\mathbf{p}}_{t}\right\|_{0, \Omega}^{2}+\left\|z_{t}-\widetilde{z}_{t}\right\|_{*}^{2} \leq C h\left(\left\|\mathbf{p}_{t}\right\|_{1 / 2, \Omega}^{2}+\left\|z_{t}\right\|_{1, \Omega}^{2}\right) .
$$

Using the above estimates we can derive the following.

Theorem 3. Let $(y, \mathbf{q}, z, \mathbf{p})$ and $\left(y_{h}(u), \mathbf{q}_{h}(u), z_{h}(u), \mathbf{p}_{h}(u)\right)$ be the solutions of (12) and (19), respectively. Then we have

$$
\mid\left\|\left(y-y_{h}(u), \mathbf{q}-\mathbf{q}_{h}(u)\right)\right\|_{+} \leq C h^{1 / 2} .
$$

Here

$$
\left\|\left(y_{h}, \mathbf{q}_{h}\right)\right\|_{+}^{2}=\left\|y_{h}\right\|_{0, \Omega}^{2}+\int_{0}^{T}\left\|y_{h}\right\|_{*}^{2} d t+\int_{0}^{T}\left\|\mathbf{q}_{h}\right\|_{0, \Omega}^{2} d t
$$

and the constant $C$ depends on the norms of $y$ and $\mathbf{q}$.
Proof. Using (12) and (19) we derive the following error equations:

$$
\begin{aligned}
\left(y_{t}-y_{h, t}(u), w_{h}\right)+B\left(\mathbf{q}-\mathbf{q}_{h}(u), w_{h}\right) & \\
+D\left(y-y_{h}(u), w_{h}\right)=0, \quad \forall w_{h} \in W_{h}, & \\
A\left(\mathbf{q}-\mathbf{q}_{h}(u), \mathbf{v}_{h}\right)-B\left(\mathbf{v}_{h}, y-y_{h}(u)\right)=0, & \\
& \forall \mathbf{v}_{h} \in \mathbf{V}_{h} .
\end{aligned}
$$

We split the errors $y-y_{h}(u)$ and $\mathbf{q}-\mathbf{q}_{h}(u)$ into

$$
\begin{aligned}
& y-y_{h}(u)=y-\tilde{y}+\tilde{y}-y_{h}(u), \\
& \mathbf{q}-\mathbf{q}_{h}(u)=\mathbf{q}-\widetilde{\mathbf{q}}+\widetilde{\mathbf{q}}-\mathbf{q}_{h}(u),
\end{aligned}
$$

where $(\tilde{y}, \widetilde{\mathbf{q}})$ is defined in (21). Then we have

$$
\begin{aligned}
\left(y_{t}-\tilde{y}_{t}, w_{h}\right)+\left(\tilde{y}_{t}-y_{h, t}(u), w_{h}\right) \\
\quad+B\left(\widetilde{\mathbf{q}}-\mathbf{q}_{h}(u), w_{h}\right)+D\left(\tilde{y}-y_{h}(u), w_{h}\right) \\
\quad+A\left(\widetilde{\mathbf{q}}-\mathbf{q}_{h}(u), \mathbf{v}_{h}\right)-B\left(\mathbf{v}_{h}, \widetilde{y}-y_{h}(u)\right)=0 .
\end{aligned}
$$

Setting $\chi=\tilde{y}-y_{h}(u)$ and $\boldsymbol{\sigma}=\widetilde{\mathbf{q}}-\mathbf{q}_{h}(u)$ gives

$$
\begin{gathered}
\left(\chi_{t}, w_{h}\right)+B\left(\boldsymbol{\sigma}, w_{h}\right)+D\left(\chi, w_{h}\right)+A\left(\boldsymbol{\sigma}, \mathbf{v}_{h}\right) \\
-B\left(\mathbf{v}_{h}, \chi\right)=-\left(y_{t}-\tilde{y}_{t}, w_{h}\right) .
\end{gathered}
$$

Choosing $w_{h}=\chi$ and $\mathbf{v}_{h}=\boldsymbol{\sigma}$ in the above equation leads to

$$
\left(\chi_{t}, \chi\right)+D(\chi, \chi)+A(\boldsymbol{\sigma}, \boldsymbol{\sigma})=-\left(y_{t}-\tilde{y}_{t}, \chi\right) \text {. }
$$

Since $D(\chi, \chi) \geq\|\chi\|_{*}^{2}$, then we deduce by Young inequality that

$$
\begin{aligned}
& \frac{1}{2} \frac{d}{d t}\|\chi\|_{0, \Omega}^{2}+\|\chi\|_{*}^{2}+\|\boldsymbol{\sigma}\|_{0, \Omega}^{2} \\
& \quad \leq \delta\|\chi\|_{0, \Omega}^{2}+C(\delta)\left\|y_{t}-\tilde{y}_{t}\right\|_{0, \Omega}^{2} .
\end{aligned}
$$

By setting $\delta$ small enough we derive

$$
\frac{1}{2} \frac{d}{d t}\|\chi\|_{0, \Omega}^{2}+\frac{1}{2}\|\chi\|_{*}^{2}+\|\boldsymbol{\sigma}\|_{0, \Omega}^{2} \leq C\left\|y_{t}-\tilde{y}_{t}\right\|_{0, \Omega}^{2}
$$

Note that $\chi(0)=0$. Integrating (49) from $0 \rightarrow t$ leads to

$$
\|\chi(t)\|_{0, \Omega}^{2}+\int_{0}^{t}\|\chi\|_{*}^{2}+\int_{0}^{t}\|\sigma\|_{0, \Omega}^{2} \leq C \int_{0}^{t}\left\|y_{t}-\tilde{y}_{t}\right\|_{0, \Omega}^{2} .
$$

Similarly, integrating (49) from $0 \rightarrow T$ yields

$$
\begin{gathered}
\|\chi(T)\|_{0, \Omega}^{2}+\int_{0}^{T}\|\chi\|_{*}^{2}+\int_{0}^{T}\|\boldsymbol{\sigma}\|_{0, \Omega}^{2} \\
\leq C \int_{0}^{T}\left\|y_{t}-\tilde{y}_{t}\right\|_{0, \Omega}^{2},
\end{gathered}
$$

which implies

$$
\int_{0}^{T}\|\chi\|_{*}^{2}+\int_{0}^{T}\|\boldsymbol{\sigma}\|_{0, \Omega}^{2} \leq C \int_{0}^{T}\left\|y_{t}-\tilde{y}_{t}\right\|_{0, \Omega}^{2} .
$$


Combining (50) and (52) we deduce that

$$
\begin{gathered}
\|\chi(t)\|_{0, \Omega}^{2}+\int_{0}^{T}\|\chi\|_{*}^{2}+\int_{0}^{T}\|\boldsymbol{\sigma}\|_{0, \Omega}^{2} \\
\leq C \int_{0}^{T}\left\|y_{t}-\tilde{y}_{t}\right\|_{0, \Omega}^{2} .
\end{gathered}
$$

By Lemma 2 we further derive

$$
\begin{gathered}
\|\chi(t)\|_{0, \Omega}^{2}+\int_{0}^{T}\|\chi\|_{*}^{2}+\int_{0}^{T}\|\boldsymbol{\sigma}\|_{0, \Omega}^{2} \\
\leq \operatorname{Ch}\left(\int_{0}^{T}\left\|\mathbf{q}_{t}\right\|_{1 / 2, \Omega}^{2}+\int_{0}^{T}\left\|y_{t}\right\|_{1, \Omega}^{2}\right) .
\end{gathered}
$$

Then by triangle inequality and Lemma 1 we obtain

$$
\begin{aligned}
& \|\|\left(y-y_{h}(u), \mathbf{q}-\mathbf{q}_{h}(u)\right) \|_{+}^{2} \leq C h\left(\|\mathbf{q}\|_{1 / 2, \Omega}^{2}+\|y\|_{1, \Omega}^{2}\right. \\
& \quad+\int_{0}^{T}\|\mathbf{q}\|_{1 / 2, \Omega}^{2}+\int_{0}^{T}\|y\|_{1, \Omega}^{2}+\int_{0}^{T}\left\|\mathbf{q}_{t}\right\|_{1 / 2, \Omega}^{2} \\
& \left.\quad+\int_{0}^{T}\left\|y_{t}\right\|_{1, \Omega}^{2}\right) .
\end{aligned}
$$

This implies the theorem result.

Theorem 4. Let $(y, \mathbf{q}, z, \mathbf{p})$ and $\left(y_{h}(u), \mathbf{q}_{h}(u), z_{h}(u), \mathbf{p}_{h}(u)\right)$ be the solutions of (12) and (19), respectively. Then we have

$$
\|\|\left(z-z_{h}(u), \mathbf{p}-\mathbf{p}_{h}(u)\right) \|\left.\right|_{+} \leq C h^{1 / 2} .
$$

Here the constant $C$ depends on the norms of $y, z$ and $\mathbf{q}, \mathbf{p}$.

Proof. Using (12) and (19) we derive the following error equations:

$$
\begin{array}{cc}
-\left(z_{t}-z_{h, t}(u), \psi_{h}\right)-B\left(\mathbf{p}-\mathbf{p}_{h}(u), \psi_{h}\right) & \\
+D\left(\psi_{h}, z-z_{h}(u)\right)=\left(y-y_{h}(u), \psi_{h}\right), & \\
\forall \psi_{h} \in W_{h} \\
A\left(\mathbf{p}-\mathbf{p}_{h}(u), \boldsymbol{\varphi}_{h}\right)+B\left(\boldsymbol{\varphi}_{h}, z-z_{h}(u)\right)=0, & \\
& \forall \boldsymbol{\varphi}_{h} \in \mathbf{V}_{h} .
\end{array}
$$

We rewrite the errors $z-z_{h}(u)$ and $\mathbf{p}-\mathbf{p}_{h}(u)$ as

$$
\begin{aligned}
& z-z_{h}(u)=z-\widetilde{z}+\widetilde{z}-z_{h}(u), \\
& \mathbf{p}-\mathbf{p}_{h}(u)=\mathbf{p}-\widetilde{\mathbf{p}}+\widetilde{\mathbf{p}}-\mathbf{p}_{h}(u),
\end{aligned}
$$

where $(\widetilde{z}, \widetilde{\mathbf{p}})$ is defined in (22). Then we have

$$
\begin{aligned}
-\left(z_{t}\right. & \left.-\widetilde{z}_{t}, \psi_{h}\right)-\left(\widetilde{z}_{t}-z_{h, t}(u), \psi_{h}\right) \\
& -B\left(\widetilde{\mathbf{p}}-\mathbf{p}_{h}(u), \psi_{h}\right)+D\left(\psi_{h}, \widetilde{z}-z_{h}(u)\right) \\
& +A\left(\widetilde{\mathbf{p}}-\mathbf{p}_{h}(u), \boldsymbol{\varphi}_{h}\right)+B\left(\boldsymbol{\varphi}_{h}, \widetilde{z}-z_{h}(u)\right) \\
= & \left(y-y_{h}(u), \psi_{h}\right) .
\end{aligned}
$$

Setting $\xi=\widetilde{z}-z_{h}(u)$ and $\boldsymbol{\rho}=\widetilde{\mathbf{p}}-\mathbf{p}_{h}(u)$ we have

$$
\begin{aligned}
& -\left(\xi_{t}, \psi_{h}\right)-B\left(\boldsymbol{\rho}, \psi_{h}\right)+D\left(\psi_{h}, \xi\right)+A\left(\boldsymbol{\rho}, \boldsymbol{\varphi}_{h}\right) \\
& \quad+B\left(\boldsymbol{\varphi}_{h}, \xi\right)=\left(z_{t}-\tilde{z}_{t}, \psi_{h}\right)+\left(y-y_{h}(u), \psi_{h}\right) .
\end{aligned}
$$

Taking $\psi_{h}=\xi$ and $\boldsymbol{\varphi}_{h}=\boldsymbol{\rho}$ yields

$$
\begin{aligned}
- & \left(\xi_{t}, \xi\right)+D(\xi, \xi)+A(\boldsymbol{\rho}, \boldsymbol{\rho}) \\
& =\left(z_{t}-\widetilde{z}_{t}, \xi\right)+\left(y-y_{h}(u), \xi\right) .
\end{aligned}
$$

Since $D(\xi, \xi) \geq\|\xi\|_{*}^{2}$, then

$$
\begin{aligned}
& -\frac{1}{2} \frac{d}{d t}\|\xi(t)\|_{0, \Omega}^{2}+\|\xi\|_{*}^{2}+\|\rho\|_{0, \Omega}^{2} \\
& \quad \leq\left|\left(z_{t}-\widetilde{z}_{t}, \xi\right)\right|+\left|\left(y-y_{h}(u), \xi\right)\right| .
\end{aligned}
$$

Using Hölder inequality and Young inequality we derive

$$
\begin{gathered}
-\frac{1}{2} \frac{d}{d t}\|\xi(t)\|_{0, \Omega}^{2}+\|\xi\|_{*}^{2}+\|\rho\|_{0, \Omega}^{2} \\
\leq \delta\|\xi\|_{0, \Omega}^{2}+C(\delta)\left\|z_{t}-\widetilde{z}_{t}\right\|_{0, \Omega}^{2} \\
+C(\delta)\left\|y-y_{h}(u)\right\|_{0, \Omega}^{2} .
\end{gathered}
$$

Choosing $\delta$ small enough we obtain

$$
\begin{aligned}
- & \frac{1}{2} \frac{d}{d t}\|\xi(t)\|_{0, \Omega}^{2}+\frac{1}{2}\|\xi\|_{*}^{2}+\|\rho\|_{0, \Omega}^{2} \\
& \leq C(\delta)\left\|z_{t}-\widetilde{z}_{t}\right\|_{0, \Omega}^{2}+C(\delta)\left\|y-y_{h}(u)\right\|_{0, \Omega}^{2} .
\end{aligned}
$$

Integrating (64) with respect to $t$ from $t \rightarrow T$ yields

$$
\begin{aligned}
& \|\xi(t)\|_{0, \Omega}^{2}+\int_{t}^{T}\|\xi\|_{*}^{2}+\int_{t}^{T}\|\rho\|_{0, \Omega}^{2} \\
& \quad \leq C \int_{0}^{T}\left\|z_{t}-\widetilde{z}_{t}\right\|_{0, \Omega}^{2}+C \int_{0}^{T}\left\|y-y_{h}(u)\right\|_{0, \Omega}^{2},
\end{aligned}
$$

where $\xi(T)=0$ was used. In an analogue way, integrating (64) from $0 \rightarrow T$ results in

$$
\begin{aligned}
& \frac{1}{2}\|\xi(0)\|_{0, \Omega}^{2}+\int_{0}^{T}\|\xi\|_{*}^{2}+\int_{0}^{T}\|\rho\|_{0, \Omega}^{2} \\
& \leq C \int_{0}^{T}\left\|z_{t}-\widetilde{z}_{t}\right\|_{0, \Omega}^{2}+C \int_{0}^{T}\left\|y-y_{h}(u)\right\|_{0, \Omega}^{2} .
\end{aligned}
$$

We further have

$$
\begin{aligned}
\int_{0}^{T}\|\xi\|_{*}^{2}+\int_{0}^{T}\|\rho\|_{0, \Omega}^{2} \leq & C \int_{0}^{T}\left\|z_{t}-\widetilde{z}_{t}\right\|_{0, \Omega}^{2} \\
& +C \int_{0}^{T}\left\|y-y_{h}(u)\right\|_{0, \Omega}^{2} .
\end{aligned}
$$

Combining (65) and (67) we deduce that

$$
\begin{aligned}
& \|\xi(t)\|_{0, \Omega}^{2}+\int_{0}^{T}\|\xi\|_{*}^{2}+\int_{0}^{T}\|\rho\|_{0, \Omega}^{2} \\
& \quad \leq C \int_{0}^{T}\left\|z_{t}-\widetilde{z}_{t}\right\|_{0, \Omega}^{2}+C \int_{0}^{T}\left\|y-y_{h}(u)\right\|_{0, \Omega}^{2} .
\end{aligned}
$$


Then we can derive the theorem result by using Lemmas 1 and 2 and Theorem 8 and triangle inequality.

Lemma 5. Let $\left(y_{h}, \mathbf{q}_{h}, z_{h}, \mathbf{p}_{h}\right)$ and $\left(y_{h}(u), \mathbf{q}_{h}(u), z_{h}(u), \mathbf{p}_{h}(u)\right)$ be the solutions of (14) and (19), respectively. Then we have

$$
\begin{gathered}
\left|\left\|\left(y_{h}-y_{h}(u), \mathbf{q}_{h}-\mathbf{q}_{h}(u)\right)\right\|\right|_{+}^{2} \leq C \int_{0}^{T}\left\|u-u_{h}\right\|_{0, \Omega}^{2}, \\
\left|\left\|\left(z_{h}-z_{h}(u), \mathbf{p}_{h}-\mathbf{p}_{h}(u)\right)\right\|\right|_{+}^{2} \leq C \int_{0}^{T}\left\|u-u_{h}\right\|_{0, \Omega}^{2} .
\end{gathered}
$$

Proof. Using (19) along with (14) leads to

$$
\begin{array}{cc}
\left(y_{h, t}-y_{h, t}(u), w_{h}\right)+B\left(\mathbf{q}_{h}-\mathbf{q}_{h}(u), w_{h}\right) & \\
+D\left(y_{h}-y_{h}(u), w_{h}\right)=\left(u_{h}-u, w_{h}\right), & \\
& \forall w_{h} \in W_{h}, \\
A\left(\mathbf{q}_{h}-\mathbf{q}_{h}(u), \mathbf{v}_{h}\right)-B\left(\mathbf{v}_{h}, y_{h}-y_{h}(u)\right)=0, &
\end{array}
$$$$
\forall \mathbf{v}_{h} \in \mathbf{V}_{h} .
$$

Setting $Y_{h}=y_{h}-y_{h}(u)$ and $\mathbf{Q}_{h}=\mathbf{q}_{h}-\mathbf{q}_{h}(u)$ and choosing $w_{h}=Y_{h}$ and $\mathbf{v}_{h}=\mathbf{Q}_{h}$ lead to

$$
\left(Y_{h, t}, Y_{h}\right)+A\left(\mathbf{Q}_{h}, \mathbf{Q}_{h}\right)+D\left(Y_{h}, Y_{h}\right)=\left(u_{h}-u, Y_{h}\right) \text {. }
$$

By Young inequality we have

$$
\begin{aligned}
& \frac{1}{2} \frac{d}{d t}\left\|Y_{h}\right\|_{0, \Omega}^{2}+\left\|\mathbf{Q}_{h}\right\|_{0, \Omega}^{2}+\left\|Y_{h}\right\|_{*}^{2} \\
& \quad \leq C(\delta)\left\|u-u_{h}\right\|_{0, \Omega}^{2}+C \delta\left\|Y_{h}\right\|_{0, \Omega}^{2} .
\end{aligned}
$$

Similar to the proof of Lemmas 1 and 2 we have

$$
\begin{gathered}
\left\|Y_{h}\right\|_{0, \Omega}^{2}+\int_{0}^{T}\left\|\mathbf{Q}_{h}\right\|_{0, \Omega}^{2}+\int_{0}^{T}\left\|Y_{h}\right\|_{*}^{2} \\
\leq C \int_{0}^{T}\left\|u-u_{h}\right\|_{0, \Omega}^{2} .
\end{gathered}
$$

By (17) and (19) we derive

$$
\begin{aligned}
& -\left(z_{h, t}-z_{h, t}(u), \psi_{h}\right)-B\left(\mathbf{p}_{h}-\mathbf{p}_{h}(u), \psi_{h}\right) \\
& \quad+D\left(\psi_{h}, z_{h}-z_{h}(u)\right)=\left(Y_{h}, \psi_{h}\right), \quad \forall \psi_{h} \in W_{h}, \\
& A\left(\mathbf{p}_{h}-\mathbf{p}_{h}(u), \boldsymbol{\varphi}_{h}\right)-B\left(\boldsymbol{\varphi}_{h}, z_{h}-z_{h}(u)\right)=0,
\end{aligned}
$$

$$
\forall \varphi_{h} \in \mathbf{V}_{h} .
$$

Setting $Z_{h}=z_{h}-z_{h}(u)$ and $\mathbf{P}_{h}=\mathbf{p}_{h}-\mathbf{p}_{h}(u)$ and choosing $\psi_{h}=Z_{h}$ and $\boldsymbol{\varphi}_{h}=\mathbf{P}_{h}$ yield

$$
-\left(Z_{h, t}, Z_{h}\right)+A\left(\mathbf{P}_{h}, \mathbf{P}_{h}\right)+D\left(Z_{h}, Z_{h}\right)=\left(Y_{h}, Z_{h}\right) .
$$

Furthermore, we derive by Young inequality

$$
\begin{gathered}
-\frac{1}{2}\left\|Z_{h}\right\|_{0, \Omega}^{2}+\left\|\mathbf{P}_{h}\right\|_{0, \Omega}^{2}+\left\|Z_{h}\right\|_{*}^{2} \\
\leq C(\delta)\left\|Y_{h}\right\|_{0, \Omega}^{2}+C \delta\left\|Z_{h}\right\|_{0, \Omega}^{2} .
\end{gathered}
$$

Similar to the proof of Lemmas 1 and 2 we have

$$
\begin{aligned}
& \left\|Z_{h}\right\|_{0, \Omega}^{2}+\int_{0}^{T}\left\|\mathbf{P}_{h}\right\|_{0, \Omega}^{2}+\int_{0}^{T}\left\|Z_{h}\right\|_{*}^{2} \leq C \int_{0}^{T}\left\|Y_{h}\right\|_{0, \Omega}^{2} \\
& \quad \leq C \int_{0}^{T}\left\|u-u_{h}\right\|_{0, \Omega}^{2} .
\end{aligned}
$$

Then the theorem result follows from (73) and (77).

Lemma 6. Assume that $(y, \mathbf{q}, z, \mathbf{p}, u)$ and $\left(y_{h}, \mathbf{q}_{h}, z_{h}, \mathbf{p}_{h}, u_{h}\right)$ be the solutions of (11) and (14), respectively. Let

$$
J_{h}^{\prime}(u)(v-u)=\int_{0}^{T}\left(\gamma u+z_{h}(u), v-u\right),
$$

where $z_{h}(u)$ is the solution of (19). Then the following estimate holds:

$$
J_{h}^{\prime}(v)(v-u)-J_{h}^{\prime}(u)(v-u) \geq \gamma\|v-u\|_{L^{2}\left(0, T ; L^{2}(\Omega)\right)}^{2} .
$$

Proof. By the definitions of $\left(y_{h}(u), \mathbf{q}_{h}(u)\right),\left(z_{h}(u), \mathbf{p}_{h}(u)\right)$ we have

$$
\begin{gathered}
\left(y_{h, t}(v)-y_{h, t}(u), w_{h}\right)+B\left(\mathbf{q}_{h}(v)-\mathbf{q}_{h}(u), w_{h}\right) \\
+D\left(y_{h}(v)-y_{h}(u), w_{h}\right)=\left(v-u, w_{h}\right), \\
\forall w_{h} \in W_{h}, \\
A\left(\mathbf{q}_{h}(v)-\mathbf{q}_{h}(u), \mathbf{v}_{h}\right)-B\left(\mathbf{v}_{h}, y_{h}(v)-y_{h}(u)\right)=0, \\
\forall \mathbf{v}_{h} \in \mathbf{V}_{h}, \\
\left(z_{h, t}(u)-z_{h, t}(v), \psi_{h}\right)-B\left(\mathbf{p}_{h}(v)-\mathbf{p}_{h}(u), \psi_{h}\right) \\
+D\left(\psi_{h}, z_{h}(v)-z_{h}(u)\right)=\left(y_{h}(v)-y_{h}(u), \psi_{h}\right), \\
A\left(\mathbf{p}_{h}(v)-\mathbf{p}_{h}(u), \boldsymbol{\varphi}_{h}\right)+B\left(\boldsymbol{\varphi}_{h}, z_{h}(v)-z_{h}(u)\right)=0 .
\end{gathered}
$$

Note that

$$
\begin{aligned}
& J_{h}^{\prime}(v)(v-u)-J_{h}^{\prime}(u)(v-u) \\
& =\int_{0}^{T}\left(\gamma v+z_{h}(v), v-u\right)-\int_{0}^{T}\left(\gamma v+z_{h}(u), v-u\right) \\
& =\int_{0}^{T}(\gamma(v-u), v-u) \\
& \quad+\int_{0}^{T}\left(z_{h}(v)-z_{h}(u), v-u\right) \\
& =\gamma\|v-u\|_{L^{2}\left(0, T ; L^{2}(\Omega)\right)}^{2} \\
& \quad+\int_{0}^{T}\left(z_{h}(v)-z_{h}(u), v-u\right) .
\end{aligned}
$$


By setting $w_{h}=z_{h}(v)-z_{h}(u), \mathbf{v}_{h}=\mathbf{p}_{h}(v)-\mathbf{p}_{h}(u)$ and $\psi_{h}=$ $y_{h}(v)-y_{h}(u), \boldsymbol{\varphi}_{h}=\mathbf{q}_{h}(v)-\mathbf{q}_{h}(u)$ in the above equations, respectively, we can prove

$$
\begin{aligned}
& \int_{0}^{T}\left(z_{h}(v)-z_{h}(u), v-u\right)=\int_{0}^{T}\left(v-u, z_{h}(v)\right. \\
& \left.\quad-z_{h}(u)\right) \\
& \quad=\int_{0}^{T}\left[D\left(y_{h}(v)-y_{h}(u), z_{h}(v)-z_{h}(u)\right)\right. \\
& +B\left(\mathbf{q}_{h}(v)-\mathbf{q}_{h}(u), z_{h}(v)-z_{h}(u)\right) \\
& +\left(y_{h}, t(v)-y_{h}, t(u), z_{h}(v)-z_{h}(u)\right) \\
& +A\left(\mathbf{q}_{h}(v)-\mathbf{q}_{h}(u), \mathbf{p}_{h}(v)-\mathbf{p}_{h}(u)\right) \\
& \left.-B\left(\mathbf{p}_{h}(v)-\mathbf{p}_{h}(u), y_{h}(v)-y_{h}(u)\right)\right] .
\end{aligned}
$$

Since $\left(y_{h}(v)-y_{h}(u)\right)(0)=0,\left(z_{h}(v)-z_{h}(u)\right)(T)=0$, and

$$
\begin{aligned}
& \int_{0}^{T}\left(y_{h, t}(v)-y_{h, t}(u), z_{h}(v)-z_{h}(u)\right) \\
& =-\int_{0}^{T}\left(y_{h}(v)-y_{h}(u), z_{h, t}(v)-z_{h, t}(u)\right) \\
& +\left.\left(y_{h}(v)-y_{h}(u), z_{h}(v)-z_{h}(u)\right)\right|_{0} ^{T}=0
\end{aligned}
$$

we can prove that

$$
\begin{aligned}
& \int_{0}^{T}\left(z_{h}(v)-z_{h}(u), v-u\right) \\
& \quad=\int_{0}^{T}\left(y_{h}(v)-y_{h}(u), y_{h}(v)-y_{h}(u)\right) \geq 0 .
\end{aligned}
$$

Therefore, we derive

$$
J_{h}^{\prime}(v)(v-u)-J_{h}^{\prime}(u)(v-u) \geq \gamma\|v-u\|_{L^{2}\left(0, T ; L^{2}(\Omega)\right)}^{2} .
$$

Lemma 7. Let $(y, \mathbf{q}, z, \mathbf{p}, u)$ and $\left(y_{h}, \mathbf{q}_{h}, z_{h}, \mathbf{p}_{h}, u_{h}\right)$ be the solutions of (11) and (14), respectively. Then we have

$$
\left\|u-u_{h}\right\|_{L^{2}\left(0, T, L^{2}(\Omega)\right)} \leq C h^{1 / 2} .
$$

Proof. By Lemma 6 we have

$$
\begin{aligned}
\gamma\left\|u-u_{h}\right\|_{L^{2}\left(0, T, L^{2}(\Omega)\right)}^{2} \leq\left(J_{h}^{\prime}(u)-J_{h}^{\prime}\left(u_{h}\right), u-u_{h}\right) \\
=\int_{0}^{T}\left(\alpha u+z_{h}(u), u-u_{h}\right) d t \\
\quad-\int_{0}^{T}\left(\alpha u_{h}+z_{h}, u-u_{h}\right) d t \\
=\int_{0}^{T}\left(\alpha u+z, u-u_{h}\right) d t \\
\quad+\int_{0}^{T}\left(z_{h}(u)-z, u-u_{h}\right) d t
\end{aligned}
$$

$$
\begin{aligned}
& +\int_{0}^{T}\left(\alpha u_{h}+z_{h}, u_{h}-u\right) d t \\
\leq & 0+\int_{0}^{T}\left(z_{h}(u)-z, u-u_{h}\right) d t+0 \\
\leq & \left\|z_{h}(u)-z\right\|_{L^{2}\left(0, T, L^{2}(\Omega)\right)}\left\|u-u_{h}\right\|_{L^{2}\left(0, T, L^{2}(\Omega)\right)} .
\end{aligned}
$$

Using the result of Theorem 4 yields

$$
\begin{aligned}
\left\|u-u_{h}\right\|_{L^{2}\left(0, T, L^{2}(\Omega)\right)} & \leq C\left\|z_{h}(u)-z\right\|_{L^{2}\left(0, T, L^{2}(\Omega)\right)} \\
& \leq C h^{1 / 2} .
\end{aligned}
$$

Theorem 8. Let $(y, \mathbf{q}, z, \mathbf{p})$ and $\left(y_{h}(u), \mathbf{q}_{h}(u), z_{h}(u), \mathbf{p}_{h}(u)\right)$ be the solutions of (12) and (14), respectively. Then we have

$$
\begin{gathered}
\left\|u-u_{h}\right\|_{L^{2}\left(0, T, L^{2}(\Omega)\right)}+\left|\left\|\left(y-y_{h}, \mathbf{q}-\mathbf{q}_{h}\right)\right\|\right|_{+} \\
+\left|\left\|\left(z-z_{h}, \mathbf{p}-\mathbf{p}_{h}\right)\right\|\right|_{+} \leq C h^{1 / 2} .
\end{gathered}
$$

Proof. By Lemma 2 and (88) we derive

$$
\begin{gathered}
\|\|\left(y-y_{h}, \mathbf{q}-\mathbf{q}_{h}\right)\left\|_{+}^{2} \leq \mid\right\|\left(y-y_{h}(u), \mathbf{q}-\mathbf{q}_{h}(u)\right) \|_{+}^{2} \\
+\mid\left\|\left(y_{h}(u)-y_{h}, \mathbf{q}_{h}(u)-\mathbf{q}_{h}\right)\right\|_{+}^{2} \leq C h\left(\|\mathbf{q}\|_{1 / 2, \Omega}^{2}\right. \\
\left.+\|y\|_{1, \Omega}^{2}\right)+C \int_{0}^{T}\left\|u-u_{h}\right\|_{0, \Omega}^{2}+C h\left(\int_{0}^{T}\|\mathbf{q}\|_{1 / 2, \Omega}^{2}\right. \\
\left.\quad+\int_{0}^{T}\|y\|_{1, \Omega}^{2}+\int_{0}^{T}\left\|\frac{\partial \mathbf{q}}{\partial t}\right\|_{1 / 2, \Omega}^{2}+\int_{0}^{T}\left\|\frac{\partial y}{\partial t}\right\|_{1, \Omega}^{2}\right) .
\end{gathered}
$$

Combining Lemma 5 and (90) yields

$$
\begin{aligned}
& \left|\left\|\left(z-z_{h}, \mathbf{p}-\mathbf{p}_{h}\right)\right\|_{+}^{2} \leq\right|\left\|\left(z-z_{h}(u), \mathbf{p}-\mathbf{p}_{h}(u)\right)\right\|_{+}^{2} \\
& +\left|\left\|\left(z_{h}(u)-z_{h}, \mathbf{p}_{h}(u)-\mathbf{p}_{h}\right)\right\|\right|_{0}^{2} \leq C h\left(\|\mathbf{p}\|_{1 / 2, \Omega}^{2}\right. \\
& \left.\quad+\|z\|_{1, \Omega}^{2}+\int_{0}^{T}\|\mathbf{p}\|_{1 / 2, \Omega}^{2}+\int_{0}^{T}\|z\|_{1, \Omega}^{2}\right) \\
& \quad+C h\left(\int_{0}^{T}\left\|\frac{\partial \mathbf{p}}{\partial t}\right\|_{1 / 2, \Omega}^{2}+\int_{0}^{T}\left\|\frac{\partial z}{\partial t}\right\|_{1, \Omega}^{2}+\int_{0}^{T}\|\mathbf{q}\|_{1 / 2, \Omega}^{2}\right. \\
& \left.\quad+\int_{0}^{T}\|y\|_{1, \Omega}^{2}\right)+C \int_{0}^{T}\left\|u-u_{h}\right\|_{0, \Omega}^{2} \cdot
\end{aligned}
$$

Combining (88)-(91) we can derive the theorem result. 


\section{Fully Discrete Error Estimate}

In this section we will prove the error estimates for the fully discrete scheme. For this purpose we firstly introduce the following auxiliary problems:

$$
\begin{aligned}
& \left(\frac{y_{h}^{n}(u)-y_{h}^{n-1}(u)}{k}, w_{h}\right)+B\left(\mathbf{q}_{h}^{n}(u), w_{h}\right) \\
& \quad+D\left(y_{h}^{n}(u), w_{h}\right)=\left(f^{n}+u^{n}, w_{h}\right), \quad \forall w_{h} \in W_{h}, \\
& A\left(\mathbf{q}_{h}^{n}(u), \mathbf{v}_{h}\right)-B\left(\mathbf{v}_{h}, y_{h}^{n}(u)\right)=0, \quad \forall \mathbf{v}_{h} \in \mathbf{V}_{h}, \\
& \left(\frac{z_{h}^{n-1}(u)-z_{h}^{n}(u)}{k}, \psi_{h}\right)-B\left(\mathbf{p}_{h}^{n-1}(u), \psi_{h}\right) \\
& \quad+D\left(\psi_{h}, z_{h}^{n-1}(u)\right)=\left(y_{h}^{n}(u)-y_{d}^{n}, \psi_{h}\right), \\
& \begin{array}{l}
A\left(\mathbf{p}_{h}^{n-1}(u), \boldsymbol{\varphi}_{h}\right)+B\left(\boldsymbol{\varphi}_{h}, z_{h}^{n-1}(u)\right)=0, \quad \forall \psi_{h} \in W_{h}, \\
y_{h}^{0}(u)=\mathbf{V}_{h},
\end{array} \\
& z_{h}^{N}(u)=0,
\end{aligned}
$$

$\forall \mathbf{x} \in \Omega$.

Lemma 9. Let $(y, \mathbf{q}, z, \mathbf{p})$ and $\left(y_{h}^{n}(u), \mathbf{q}_{h}^{n}(u), z_{h}^{n-1}(u), \mathbf{p}_{h}^{n-1}(u)\right)$ be the solutions of (12) and (92), respectively. Then we have

$$
\left\|\left(y-y_{h}(u), \mathbf{q}-\mathbf{q}_{h}(u)\right)\right\|_{\diamond} \leq C\left(h^{1 / 2}+k\right),
$$

where

$$
\mid\|(y, \mathbf{q})\|_{\diamond}^{2}=\left\|y^{n}\right\|_{0, \Omega}^{2}+k \sum_{n=1}^{N}\left\|y^{n}\right\|_{*}^{2}+k \sum_{n=1}^{N}\left\|\mathbf{q}^{n}\right\|_{0, \Omega}^{2} .
$$

Proof. Using (12) and (92) we derive the following error equations:

$$
\begin{aligned}
& \left(\frac{\partial y^{n}}{\partial t}-\frac{y_{h}^{n}(u)-y_{h}^{n-1}(u)}{k}, w_{h}\right)+B\left(\mathbf{q}^{n}-\mathbf{q}_{h}^{n}(u), w_{h}\right) \\
& \quad+D\left(y^{n}-y_{h}^{n}(u), w_{h}\right)=0, \quad \forall w_{h} \in W_{h} \\
& A\left(\mathbf{q}^{n}-\mathbf{q}_{h}^{n}(u), \mathbf{v}_{h}\right)-B\left(\mathbf{v}_{h}, y^{n}-y_{h}^{n}(u)\right)=0
\end{aligned}
$$$$
\forall \mathbf{q} \in \mathbf{V}_{\mathbf{h}} .
$$

Setting $\chi=\tilde{y}-y_{h}(u), \rho=y-\tilde{y}$, and $\boldsymbol{\sigma}=\widetilde{\mathbf{q}}-\mathbf{q}_{h}(u)$ and combining the definition of $\tilde{y}$ and $\widetilde{\mathbf{q}}$ we have

$$
\begin{aligned}
& \left(\frac{\chi^{n}-\chi^{n-1}}{k}, w_{h}\right)+B\left(\boldsymbol{\sigma}^{n}, w_{h}\right)+D\left(\chi^{n}, w_{h}\right) \\
& \quad=-\left(\frac{\partial y^{n}}{\partial t}-\frac{y^{n}-y^{n-1}}{k}, w_{h}\right)-\left(\frac{\rho^{n}-\rho^{n-1}}{k}, w_{h}\right), \\
& A\left(\boldsymbol{\sigma}^{n}, \mathbf{v}_{h}\right)-B\left(\mathbf{v}_{h}, \chi^{n}\right)=0 .
\end{aligned}
$$

Testing (96) with $w_{h}=\chi^{n}$ and $\mathbf{v}_{h}=\boldsymbol{\sigma}^{n}$ yields

$$
\begin{aligned}
& \left(\frac{\chi^{n}-\chi^{n-1}}{k}, \chi^{n}\right)+A\left(\boldsymbol{\sigma}^{n}, \boldsymbol{\sigma}^{n}\right)+D\left(\chi^{n}, \chi^{n}\right) \\
& =-\left(\frac{\partial y^{n}}{\partial t}-\frac{y^{n}-y^{n-1}}{k}, \chi^{n}\right)-\left(\frac{\rho^{n}-\rho^{n-1}}{k}, \chi^{n}\right) \\
& =D_{1}+D_{2} .
\end{aligned}
$$

By Taylor expansion with integral reminder we deduce that

$$
\begin{aligned}
\frac{\partial y^{n}}{\partial t}-\frac{y^{n}-y^{n-1}}{k} & =\frac{1}{k} \int_{t_{n-1}}^{t_{n}}\left(t-t_{n-1}\right) \frac{\partial^{2} y}{\partial t^{2}} d t, \\
\rho^{n}-\rho^{n-1} & =\int_{t_{n-1}}^{t_{n}} \frac{\partial \rho}{\partial t} d t .
\end{aligned}
$$

By Hölder inequality we obtain

$$
\begin{gathered}
\left\|\frac{\partial y^{n}}{\partial t}-\frac{y^{n}-y^{n-1}}{k}\right\|_{0, \Omega}^{2} \leq k \int_{t_{n-1}}^{t_{n}}\left\|\frac{\partial^{2} y}{\partial t^{2}}\right\|_{0, \Omega}^{2} d t, \\
\left\|\rho^{n}-\rho^{n-1}\right\|_{0, \Omega}^{2} \leq k \int_{t_{n-1}}^{t_{n}}\left\|\frac{\partial \rho}{\partial t}\right\|_{0, \Omega}^{2} d t .
\end{gathered}
$$

Then by Young inequality we derive

$$
\begin{aligned}
& D_{1} \leq C(\delta) k \int_{t_{n-1}}^{t_{n}}\left\|\frac{\partial^{2} y}{\partial t^{2}}\right\|_{0, \Omega}^{2} d t+\delta\left\|\chi^{n}\right\|_{0, \Omega}^{2}, \\
& D_{2} \leq C(\delta) \frac{1}{k} \int_{t_{n-1}}^{t_{n}}\left\|\frac{\partial \rho}{\partial t}\right\|_{0, \Omega}^{2} d t+\delta\left\|\chi^{n}\right\|_{0, \Omega}^{2} .
\end{aligned}
$$

Choosing $\delta$ to be small enough leads to

$$
\begin{aligned}
& \frac{1}{2 k}\left(\left\|\chi^{n}\right\|_{0, \Omega}^{2}-\left\|\chi^{n-1}\right\|_{0, \Omega}^{2}\right)+\left\|\sigma^{n}\right\|_{0, \Omega}^{2}+\frac{1}{2}\left\|\chi^{n}\right\|_{*}^{2} \\
& \leq C k \int_{t_{n-1}}^{t_{n}}\left\|\frac{\partial^{2} y}{\partial t^{2}}\right\|_{0, \Omega}^{2} d t+C \frac{1}{k} \int_{t_{n-1}}^{t_{n}}\left\|\frac{\partial \rho}{\partial t}\right\|_{0, \Omega}^{2} d t .
\end{aligned}
$$

Multiplying (114) by $2 k$ and summing up with respect to $n$ from 1 to $m$ yield

$$
\begin{aligned}
& \left\|\chi^{m}\right\|_{0, \Omega}^{2}+\tau \sum_{n=1}^{m}\left\|\boldsymbol{\sigma}^{n}\right\|_{0, \Omega}^{2}+\tau \sum_{n=1}^{m}\left\|\chi^{n}\right\|_{*}^{2} \\
& \leq C k^{2} \int_{0}^{t_{m}}\left\|\frac{\partial^{2} y}{\partial t^{2}}\right\|_{0, \Omega}^{2} d t+C \int_{0}^{t_{m}}\left\|\frac{\partial \rho}{\partial t}\right\|_{0, \Omega}^{2} d t .
\end{aligned}
$$

Multiplying (101) by $2 k$ and summing up with respect to $n$ from 1 to $N$ lead to

$$
\begin{aligned}
& \left\|\chi^{N}\right\|_{0, \Omega}^{2}+k \sum_{n=1}^{N}\left\|\boldsymbol{\sigma}^{n}\right\|_{0, \Omega}^{2}+k \sum_{n=1}^{N}\left\|\chi^{n}\right\|_{*}^{2} \\
& \leq C k^{2} \int_{0}^{T}\left\|\frac{\partial^{2} y}{\partial t^{2}}\right\|_{0, \Omega}^{2} d t+C \int_{0}^{T}\left\|\frac{\partial \rho}{\partial t}\right\|_{0, \Omega}^{2} d t .
\end{aligned}
$$


Collecting above estimates we have

$$
\begin{aligned}
& \left\|\chi^{m}\right\|_{0, \Omega}^{2}+k \sum_{n=1}^{N}\left\|\sigma^{n}\right\|_{0, \Omega}^{2}+k \sum_{n=1}^{N}\left\|\chi^{n}\right\|_{*}^{2} \\
& \leq C k^{2} \int_{0}^{T}\left\|\frac{\partial^{2} y}{\partial t^{2}}\right\|_{0, \Omega}^{2} d t+C \int_{0}^{T}\left\|\frac{\partial \rho}{\partial t}\right\|_{0, \Omega}^{2} d t .
\end{aligned}
$$

Using above estimates, Lemmas 1 and 2, and triangle inequality we deduce that

$$
\begin{aligned}
& \|\|\left(y-y_{h}(u), \mathbf{q}-\mathbf{q}_{h}(u)\right) \|_{\diamond} \\
& \quad \leq C h^{1 / 2}\left(\|\mathbf{q}\|_{L^{\infty}\left(0, T ; H^{1 / 2}(\Omega)\right)}+\|y\|_{L^{\infty}\left(0, T ; H^{1}(\Omega)\right)}\right. \\
& \left.\quad+\left\|\mathbf{q}_{t}\right\|_{L^{2}\left(0, T ; H^{1 / 2}(\Omega)\right)}+\left\|y_{t}\right\|_{L^{2}\left(0, T ; H^{1}(\Omega)\right)}\right) \\
& \quad+C k\left\|\frac{\partial^{2} y}{\partial t^{2}}\right\|_{L^{2}\left(0, T ; L^{2}(\Omega)\right)} .
\end{aligned}
$$

Lemma 10. Let $(y, \mathbf{q}, z, \mathbf{p})$ and $\left(y_{h}^{n}(u), \mathbf{q}_{h}^{n}(u), z_{h}^{n-1}(u)\right.$, $\left.\mathbf{p}_{h}^{n-1}(u)\right)$ be the solutions of (12) and (92), respectively. Then we have

$$
\left\|\left(z-z_{h}(u), \mathbf{p}-\mathbf{p}_{h}(u)\right)\right\|_{\diamond} \leq C\left(h^{1 / 2}+k\right) .
$$

Proof. Using (12) and (92) we derive the following error equations:

$$
\begin{aligned}
& \left(-\frac{z_{h}^{n-1}(u)-z_{h}^{n}(u)}{k}-\frac{\partial z^{n-1}}{\partial t}, \psi_{h}\right) \\
& \quad-B\left(\mathbf{p}^{n-1}-\mathbf{p}_{h}^{n-1}(u), \psi_{h}\right) \\
& \quad+D\left(\psi_{h}, z^{n-1}-z_{h}^{n-1}(u)\right)=-\left(y_{h}^{n}(u)-y^{n-1}, \psi_{h}\right) \\
& \quad+\left(y_{d}^{n}-y_{d}^{n-1}, \psi_{h}\right), \quad \forall \psi_{h} \in W_{h}, \\
& A\left(\mathbf{p}^{n-1}-\mathbf{p}_{h}^{n-1}(u), \mathbf{v}_{h}\right) \\
& \quad+B\left(\mathbf{v}_{h}, z^{n-1}-z_{h}^{n-1}(u)\right)=0, \quad \forall \mathbf{v}_{h} \in \mathbf{V}_{h} .
\end{aligned}
$$

Setting $\xi=\widetilde{z}-z_{h}(u), \eta=z-\widetilde{z}$, and $\boldsymbol{\sigma}=\widetilde{\mathbf{p}}-\mathbf{p}_{h}(u)$ and using the definitions of $\widetilde{z}$ and $\widetilde{\mathbf{p}}$ give

$$
\begin{gathered}
\left(\frac{\xi^{n-1}-\xi^{n}}{k}, \psi_{h}\right)+B\left(\boldsymbol{\sigma}^{n-1}, \psi_{h}\right)+D\left(\psi_{h}, \xi^{n-1}\right) \\
=-\left(y_{h}^{n}(u)-y^{n-1}, \psi_{h}\right)+\left(y_{d}^{n}-y_{d}^{n-1}, \psi_{h}\right) \\
\quad+\left(\frac{\partial z^{n-1}}{\partial t}+\frac{z^{n-1}-z^{n}}{k}, \xi^{n-1}\right) \\
\quad-\left(\frac{\eta^{n-1}-\eta^{n}}{k}, \xi^{n-1}\right), \\
A\left(\boldsymbol{\sigma}^{n-1}, \mathbf{v}_{h}\right)-B\left(\mathbf{v}_{h}, \xi^{n-1}\right)=0, \quad \forall \mathbf{v}_{h} \in \mathbf{V}_{h} .
\end{gathered}
$$

Choosing $w_{h}=\xi^{n-1}$ and $\mathbf{v}_{h}=\boldsymbol{\sigma}^{n}$ in (108) yields

$$
\begin{aligned}
& \left(\frac{\xi^{n-1}-\xi^{n}}{k}, \xi^{n-1}\right)+A\left(\boldsymbol{\sigma}^{n-1}, \boldsymbol{\sigma}^{n-1}\right)+D\left(\xi^{n-1}, \xi^{n-1}\right) \\
& =-\left(y_{h}^{n}(u)-y^{n-1}, \xi^{n-1}\right)+\left(y_{d}^{n}-y_{d}^{n-1}, \xi^{n-1}\right) \\
& \quad+\left(\frac{\partial z^{n-1}}{\partial t}+\frac{z^{n-1}-z^{n}}{k}, \xi^{n-1}\right) \\
& \quad-\left(\frac{\eta^{n-1}-\eta^{n}}{k}, \xi^{n-1}\right)=\sum_{i=1}^{4} T_{i} .
\end{aligned}
$$

Using Hölder inequality and Young inequality we obtain

$$
\begin{aligned}
& T_{1} \leq C(\delta)\left(\left\|y_{h}^{n}(u)-y^{n}\right\|_{0, \Omega}^{2}+k \int_{t_{n-1}}^{t_{n}}\left\|\frac{\partial y}{\partial t}\right\|_{0, \Omega}^{2} d t\right) \\
&+\delta\left\|\xi^{n-1}\right\|_{0, \Omega}^{2}, \\
& T_{2} \leq C(\delta) k \int_{t_{n-1}}^{t_{n}}\left\|\frac{\partial y_{d}}{\partial t}\right\|_{0, \Omega}^{2} d t+\delta\left\|\xi^{n-1}\right\|_{0, \Omega}^{2}, \\
& T_{3} \leq C(\delta) k \int_{t_{n-1}}^{t_{n}}\left\|\frac{\partial^{2} z}{\partial t^{2}}\right\|_{0, \Omega}^{2} d t+\delta\left\|\xi^{n-1}\right\|_{0, \Omega}^{2}, \\
& T_{4} \leq C(\delta) \frac{1}{k} \int_{t_{n-1}}^{t_{n}}\left\|\frac{\partial \eta}{\partial t}\right\|_{0, \Omega}^{2} d t+\delta\left\|\xi^{n-1}\right\|_{0, \Omega}^{2} .
\end{aligned}
$$

Then by setting $\delta$ small enough we have

$$
\begin{aligned}
& \frac{1}{2 k}\left(\left\|\xi^{n-1}\right\|_{0, \Omega}^{2}-\left\|\xi^{n}\right\|_{0, \Omega}^{2}\right)+\left\|\sigma^{n-1}\right\|_{0, \Omega}^{2}+\left\|\xi^{n-1}\right\|_{*}^{2} \\
& \leq C\left\|y_{h}^{n}(u)-y^{n}\right\|_{0, \Omega}^{2}+C k \int_{t_{n-1}}^{t_{n}}\left\|\frac{\partial y}{\partial t}\right\|_{0, \Omega}^{2} d t \\
& \quad+C k \int_{t_{n-1}}^{t_{n}}\left\|\frac{\partial y_{d}}{\partial t}\right\|_{0, \Omega}^{2} d t+C k \int_{t_{n-1}}^{t_{n}}\left\|\frac{\partial^{2} z}{\partial t^{2}}\right\|_{0, \Omega}^{2} d t \\
& +C \frac{1}{k} \int_{t_{n-1}}^{t_{n}}\left\|\frac{\partial \eta}{\partial t}\right\|_{0, \Omega}^{2} d t .
\end{aligned}
$$

Multiplying (111) by $2 k$ and summing up with respect to $n$ from $m$ to $N$ yield

$$
\begin{gathered}
\left\|\xi^{m-1}\right\|_{0, \Omega}^{2}+k \sum_{n=m}^{N}\left\|\boldsymbol{\sigma}^{n-1}\right\|_{0, \Omega}^{2}+k \sum_{n=m}^{N}\left\|\xi^{n-1}\right\|_{*}^{2} \\
\leq C k \sum_{n=m}^{N}\left\|y_{h}^{n}(u)-y^{n}\right\|_{0, \Omega}^{2}+C k^{2} \int_{t_{m}}^{T}\left\|\frac{\partial y}{\partial t}\right\|_{0, \Omega}^{2} d t \\
+C k^{2} \int_{t_{m}}^{T}\left\|\frac{\partial y_{d}}{\partial t}\right\|_{0, \Omega}^{2} d t+C k^{2} \int_{t_{m}}^{T}\left\|\frac{\partial^{2} z}{\partial t^{2}}\right\|_{0, \Omega}^{2} d t \\
+C \int_{t_{m}}^{T}\left\|\frac{\partial \eta}{\partial t}\right\|_{0, \Omega}^{2} d t .
\end{gathered}
$$


Multiplying (111) by $2 k$ and summing up with respect to $n$ from 1 to $N$ give

$$
\begin{aligned}
& \left\|\xi^{0}\right\|_{0, \Omega}^{2}+k \sum_{n=1}^{N}\left\|\boldsymbol{\sigma}^{n-1}\right\|_{0, \Omega}^{2}+k \sum_{n=1}^{N}\left\|\xi^{n-1}\right\|_{*}^{2} \\
& \leq C k \sum_{n=1}^{N}\left\|y_{h}^{n}(u)-y^{n}\right\|_{0, \Omega}^{2}+C k^{2} \int_{0}^{T}\left\|\frac{\partial y}{\partial t}\right\|_{0, \Omega}^{2} d t \\
& +C k^{2} \int_{0}^{T}\left\|\frac{\partial y_{d}}{\partial t}\right\|_{0, \Omega}^{2} d t+C k^{2} \int_{0}^{T}\left\|\frac{\partial^{2} z}{\partial t^{2}}\right\|_{0, \Omega}^{2} d t \\
& +C \int_{0}^{T}\left\|\frac{\partial \eta}{\partial t}\right\|_{0, \Omega}^{2} d t .
\end{aligned}
$$

Collecting (112) and (113) we obtain

$$
\begin{aligned}
& \left\|\xi^{n-1}\right\|_{0, \Omega}^{2}+k \sum_{n=1}^{N}\left\|\boldsymbol{\sigma}^{n-1}\right\|_{0, \Omega}^{2}+k \sum_{n=1}^{N}\left\|\xi^{n-1}\right\|_{*}^{2} \\
& \leq C k \sum_{n=1}^{N}\left\|y_{h}^{n}(u)-y^{n}\right\|_{0, \Omega}^{2}+C k^{2} \int_{0}^{T}\left\|\frac{\partial y}{\partial t}\right\|_{0, \Omega}^{2} d t \\
& +C k^{2} \int_{0}^{T}\left\|\frac{\partial y_{d}}{\partial t}\right\|_{0, \Omega}^{2} d t+C k^{2} \int_{0}^{T}\left\|\frac{\partial^{2} z}{\partial t^{2}}\right\|_{0, \Omega}^{2} d t \\
& +C \int_{0}^{T}\left\|\frac{\partial \eta}{\partial t}\right\|_{0, \Omega}^{2} d t .
\end{aligned}
$$

By Lemmas 1 and 2 and triangle inequality we derive

$$
\begin{aligned}
& \|\|\left(z-z_{h}(u), \mathbf{p}-\mathbf{p}_{h}(u)\right) \|_{\diamond} \\
& \quad \leq C h^{1 / 2}\left(\|\mathbf{q}\|_{L^{\infty}\left(0, T ; H^{1 / 2}(\Omega)\right)}+\|y\|_{L^{\infty}\left(0, T ; H^{1}(\Omega)\right)}\right. \\
& \left.\quad+\left\|\mathbf{q}_{t}\right\|_{L^{2}\left(0, T ; H^{1 / 2}(\Omega)\right)}+\left\|y_{t}\right\|_{L^{2}\left(0, T ; H^{1}(\Omega)\right)}\right) \\
& \quad+C h^{1 / 2}\left(\|\mathbf{p}\|_{L^{\infty}\left(0, T ; H^{1 / 2}(\Omega)\right)}+\|z\|_{L^{\infty}\left(0, T ; H^{1}(\Omega)\right)}\right. \\
& \left.\quad+\left\|\mathbf{p}_{t}\right\|_{L^{2}\left(0, T ; H^{1 / 2}(\Omega)\right)}+\left\|z_{t}\right\|_{L^{2}\left(0, T ; H^{1}(\Omega)\right)}\right) \\
& \quad+C k\left(\left\|\frac{\partial y}{\partial t}\right\|_{L^{2}\left(0, T ; L^{2}(\Omega)\right)}+\left\|\frac{\partial y_{d}}{\partial t}\right\|_{L^{2}\left(0, T ; L^{2}(\Omega)\right)}\right. \\
& \left.\quad+\left\|\frac{\partial^{2} z}{\partial t^{2}}\right\|_{L^{2}\left(0, T ; L^{2}(\Omega)\right)}\right) .
\end{aligned}
$$

Lemma 11. Let $\left(y_{h}^{n}, \mathbf{q}_{h}^{n}, z_{h}^{n-1}, \mathbf{p}_{h}^{n-1}\right)$ and $\left(y_{h}^{n}(u), \mathbf{q}_{h}^{n}(u), z_{h}^{n-1}(u)\right.$, $\left.\mathbf{p}_{h}^{n-1}(u)\right)$ be the solutions of (12) and (92), respectively. Then we have

$$
\begin{aligned}
& \mid\left\|\left(y_{h}-y_{h}(u), \mathbf{q}_{h}-\mathbf{q}_{h}(u)\right)\right\|_{\diamond}^{2} \\
& \quad \leq C k \sum_{n=1}^{N}\left\|u_{h}^{n}-u^{n}\right\|_{0, \Omega}^{2}, \\
& \mid\left\|\left(z_{h}-z_{h}(u), \mathbf{p}_{h}-\mathbf{p}_{h}(u)\right)\right\|_{\diamond}^{2} \leq C k \sum_{n=1}^{N}\left\|u_{h}^{n}-u^{n}\right\|_{0, \Omega}^{2} .
\end{aligned}
$$

Proof. Using (92) along with (17) leads to

$$
\begin{aligned}
& \left(\frac{y_{h}^{n}-y_{h}^{n}(u)-\left(y_{h}^{n-1}-y_{h}^{n-1}(u)\right)}{k}, w_{h}\right) \\
& \quad+B\left(\mathbf{q}_{h}^{n}-\mathbf{q}_{h}^{n}(u), w_{h}\right) \\
& \quad+D\left(y_{h}^{n}-y_{h}^{n}(u), w_{h}\right)=\left(u_{h}^{n}-u^{n}, w_{h}\right), \\
& A\left(\mathbf{q}_{h}^{n}-\mathbf{q}_{h}^{n}(u), \mathbf{v}_{h}\right)-B\left(\mathbf{v}_{h}, y_{h}^{n}-y_{h}^{n}(u)\right)=0 .
\end{aligned}
$$

Choosing $w_{h}=y_{h}^{n}-y_{h}^{n}(u)$ and $\mathbf{v}_{h}=\mathbf{q}_{h}^{n}-\mathbf{q}_{h}^{n}(u)$ in the above equations and adding the resulting equations together yield

$$
\begin{aligned}
& \left(\frac{y_{h}^{n}-y_{h}^{n}(u)-\left(y_{h}^{n-1}-y_{h}^{n-1}(u)\right)}{k}, y_{h}^{n}-y_{h}^{n}(u)\right) \\
& \quad+B\left(\mathbf{q}_{h}^{n}-\mathbf{q}_{h}^{n}(u), y_{h}^{n}-y_{h}^{n}(u)\right) \\
& \quad+D\left(y_{h}^{n}-y_{h}^{n}(u), y_{h}^{n}-y_{h}^{n}(u)\right) \\
& \quad+A\left(\mathbf{q}_{h}^{n}-\mathbf{q}_{h}^{n}(u), \mathbf{q}_{h}^{n}-\mathbf{q}_{h}^{n}(u)\right) \\
& \quad-B\left(\mathbf{q}_{h}^{n}-\mathbf{q}_{h}^{n}(u), y_{h}^{n}-y_{h}^{n}(u)\right) \\
& =\left(u_{h}^{n}-u^{n}, y_{h}^{n}-y_{h}^{n}(u)\right) .
\end{aligned}
$$

Setting $\mathscr{Y}_{h}=y_{h}-y_{h}(u)$ and $\mathbb{Q}_{h}=\mathbf{q}_{h}-\mathbf{q}_{h}(u)$ in the above equation leads to

$$
\begin{aligned}
& \left(\frac{\mathscr{Y}_{h}^{n}-\mathscr{Y}_{h}^{n-1}}{k}, \mathscr{Y}_{h}^{n}\right)+D\left(\mathscr{Y}_{h}^{n}, \mathscr{Y}_{h}^{n}\right)+A\left(\widehat{Q}_{h}^{n}, \widehat{Q}_{h}^{n}\right) \\
& \quad=\left(u_{h}^{n}-u^{n}, \mathscr{Y}_{h}^{n}\right),
\end{aligned}
$$

and then

$$
\begin{aligned}
& \left(\frac{\mathscr{Y}_{h}^{n}-\mathscr{Y}_{h}^{n-1}}{k}, \mathscr{Y}_{h}^{n}\right)+\left\|\mathscr{Y}_{h}^{n}\right\|_{*}^{2}+\left\|\widehat{Q}_{h}^{n}\right\|_{0, \Omega}^{2} \\
& \leq\left(u_{h}^{n}-u^{n}, \mathscr{Y}_{h}^{n}\right) .
\end{aligned}
$$

Furthermore, we have

$$
\begin{aligned}
& \frac{1}{2 k}\left(\left\|\mathscr{Y}_{h}^{n}\right\|_{0, \Omega}^{2}-\left\|\mathscr{Y}_{h}^{n-1}\right\|_{0, \Omega}^{2}\right)+\left\|\mathscr{Y}_{h}^{n}\right\|_{*}^{2}+\left\|\boldsymbol{Q}_{h}^{n}\right\|_{0, \Omega}^{2} \\
& \quad \leq C(\delta)\left\|u_{h}^{n}-u^{n}\right\|_{0, \Omega}^{2}+\delta\left\|\mathscr{Y}_{h}^{n}\right\|_{0, \Omega}^{2} .
\end{aligned}
$$

Similar to the proof of Lemmas 9 and 10 we have

$$
\begin{aligned}
& \left\|\mathscr{Y}_{h}^{n}\right\|_{0, \Omega}^{2}+k \sum_{n=1}^{N}\left\|\mathscr{Y}_{h}^{n}\right\|_{*}^{2}+k \sum_{n=1}^{N}\left\|\mathscr{Q}_{h}^{n}\right\|_{0, \Omega}^{2} \\
& \leq C k \sum_{n=1}^{N}\left\|u_{h}^{n}-u^{n}\right\|_{0, \Omega}^{2}
\end{aligned}
$$


Similarly, by (17) and (92) we derive

$$
\begin{aligned}
& \left(\frac{z_{h}^{n-1}-z_{h}^{n-1}(u)-\left(z_{h}^{n}-z_{h}^{n}(u)\right)}{k}, \psi_{h}\right) \\
& \quad-B\left(\mathbf{p}_{h}^{n-1}-\mathbf{p}_{h}^{n-1}(u), \psi_{h}\right) \\
& \quad+D\left(\psi_{h}, z_{h}^{n-1}-z_{h}^{n-1}(u)\right) \\
& \quad=\left(y_{h}^{n-1}-y_{h}^{n-1}(u), \psi_{h}\right), \\
& A\left(\mathbf{p}_{h}^{n-1}-\mathbf{p}_{h}^{n-1}(u), \varphi_{h}\right)+B\left(\varphi_{h}, z_{h}^{n-1}-z_{h}^{n-1}(u)\right) \\
& \quad=0 .
\end{aligned}
$$

Taking $\psi_{h}=z_{h}^{n-1}-z_{h}^{n-1}(u)$ and $\varphi_{h}=\mathbf{p}_{h}^{n-1}-\mathbf{p}_{h}^{n-1}(u)$ in the above equation, setting $\mathscr{Z}_{h}^{n-1}=z_{h}^{n-1}-z_{h}^{n-1}(u)$ and $\mathscr{P}_{h}^{n-1}=$ $p_{h}^{n-1}-p_{h}^{n-1}(u)$, and using similar argument to (124) give

$$
\begin{gathered}
\left\|\mathscr{E}_{h}^{n-1}\right\|_{0, \Omega}^{2}+k \sum_{n=1}^{N}\left\|\mathscr{Z}_{h}^{n-1}\right\|_{*}^{2}+k \sum_{n=1}^{N}\left\|\mathscr{P}_{h}^{n-1}\right\|_{0, \Omega}^{2} \\
\leq C k \sum_{n=1}^{N}\left\|y_{h}^{n}-y_{h}^{n}(u)\right\|_{0, \Omega}^{2} \leq C k \sum_{n=1}^{N}\left\|u_{h}^{n}-u^{n}\right\|_{0, \Omega}^{2} .
\end{gathered}
$$

Then the theorem result follows from (122) and (124).

Lemma 12. Let $(y, \mathbf{q}, z, \mathbf{p}, u)$ and $\left(y_{h}^{n}, \mathbf{q}_{h}^{n}, z_{h}^{n-1}, \mathbf{p}_{h}^{n-1}, u_{h}^{n}\right)$ be the solutions of (12) and (92), respectively. Let

$$
\widehat{J}_{h}^{\prime}(u)(v-u)=k \sum_{n=1}^{N}\left(\gamma u^{n}+z_{h}^{n-1}(u), v^{n}-u^{n}\right),
$$

where $z_{h}^{n-1}(u)$ is the solution of (92). Then the following estimate holds:

$$
\left(\widehat{J}_{h}^{\prime}(v)-\widehat{J}_{h}^{\prime}(u), v-u\right) \geq \gamma|\|v-u\||_{L^{2}\left(0, T, L^{2}(\Omega)\right)}^{2} .
$$

Here $|\|v-u\||_{L^{2}\left(0, T ; L^{2}(\Omega)\right)}^{2}:=k \sum_{n=1}^{N}\left\|v^{n}-u^{n}\right\|^{2}$.

Proof. Note that

$$
\begin{aligned}
& \left(\widehat{J}_{h}^{\prime}(v)-\widehat{J}_{h}^{\prime}(u), v-u\right) \\
& =k \sum_{n=1}^{N}\left(\gamma v^{n}+z_{h}^{n-1}(v)-\gamma u^{n}-z_{h}^{n-1}(u), v^{n}-u^{n}\right) \\
& =k \sum_{n=1}^{N}\left(\gamma v^{n}-\gamma u^{n}, v^{n}-u^{n}\right) \\
& \quad+k \sum_{n=1}^{N}\left(z_{h}^{n-1}(v)-z_{h}^{n-1}(u), v^{n}-u^{n}\right) \\
& =\gamma|\|v-u\||_{L^{2}\left(0, T, L^{2}(\Omega)\right)}^{2} \\
& \quad+k \sum_{n=1}^{N}\left(z_{h}^{n-1}(v)-z_{h}^{n-1}(u), v^{n}-u^{n}\right) .
\end{aligned}
$$

Setting $Y_{h}^{n}=y_{h}^{n}(v)-y_{h}^{n}(u), \mathbf{Q}_{h}^{n}=\mathbf{q}_{h}^{n}(v)-\mathbf{q}_{h}^{n}(u), Z_{h}^{n-1}=$ $z_{h}^{n-1}(v)-z_{h}^{n-1}(u)$, and $\mathbf{P}_{h}^{n-1}=\mathbf{p}_{h}^{n-1}(v)-\mathbf{p}_{h}^{n-1}(u)$, by the definition of $y_{h}^{n}(u)$ and $z_{h}^{n-1}(u)$ we have

$$
\begin{aligned}
& \left(\frac{Y_{h}^{n}-Y_{h}^{n-1}}{\tau}, w_{h}\right)+B\left(\mathbf{Q}_{h}^{n}, w_{h}\right)+D\left(Y_{h}^{n}, w_{h}\right) \\
& \quad=\left(v^{n}-u^{n}, w_{h}\right), \\
& A\left(\mathbf{Q}_{h}^{n}, \mathbf{v}_{h}\right)-B\left(\mathbf{v}_{h}, Y_{h}^{n}\right)=0, \\
& \left(\frac{Z_{h}^{n-1}-Z_{h}^{n}}{\tau}, \psi_{h}\right)-B\left(\mathbf{P}_{h}^{n}, \psi_{h}\right)+D\left(\psi_{h}, Z_{h}^{n-1}\right) \\
& \quad=\left(Y_{h}^{n}, \psi_{h}\right), \\
& A\left(\mathbf{P}_{h}^{n-1}, \boldsymbol{\varphi}_{h}\right)+B\left(\boldsymbol{\varphi}_{h}, Z_{h}^{n-1}\right)=0 .
\end{aligned}
$$

Choosing $w_{h}=Z_{h}^{n-1}, \mathbf{v}_{h}=\mathbf{P}_{h}^{n-1}$ and $\psi_{h}=Y_{h}^{n}, \boldsymbol{\varphi}_{h}=\mathbf{Q}_{h}^{n}$ in the above equations, respectively, we can easily prove that

$$
k \sum_{n=1}^{N}\left(z_{h}^{n-1}(v)-z_{h}^{n-1}(u), v^{n}-u^{n}\right) \geq 0
$$

Therefore, we derive

$$
\left(\widehat{J}_{h}^{\prime}(v)-\widehat{J}_{h}^{\prime}(u), v-u\right) \geq \gamma|\|v-u\||_{L^{2}\left(0, T, L^{2}(\Omega)\right)}^{2} .
$$

Lemma 13. Let $(y, \mathbf{q}, z, \mathbf{p}, u)$ and $\left(y_{h}^{n}, \mathbf{q}_{h}^{n}, z_{h}^{n-1}, \mathbf{p}_{h}^{n-1}, u_{h}^{n}\right)$ be the solutions of (12) and (92), respectively. Then we have

$$
\left|\left\|u-u_{h}\right\|\right|_{L^{2}\left(0, T, L^{2}(\Omega)\right)} \leq C h^{1 / 2}+C k\left\|\frac{\partial z}{\partial t}\right\|_{L^{2}\left(0, T, L^{2}(\Omega)\right)} .
$$

Proof. Following Lemma 12 we derive

$$
\begin{aligned}
\gamma\left|\left\|u-u_{h}\right\|\right|_{L^{2}\left(0, T, L^{2}(\Omega)\right)}^{2} \leq\left(\widehat{J}_{h}^{\prime}(u)-\widehat{J}_{h}^{\prime}\left(u_{h}\right), u-u_{h}\right) \\
=k \sum_{n=1}^{N}\left(\gamma u^{n}+z_{h}^{n-1}(u), u^{n}-u_{h}^{n}\right)_{U} \\
\quad-k \sum_{n=1}^{N}\left(\gamma u^{n}+z_{h}^{n-1}, u^{n}-u_{h}^{n}\right) \\
=k \sum_{n=1}^{N}\left(\gamma u^{n}+z^{n}, u^{n}-u_{h}^{n}\right)_{U} \\
+k \sum_{n=1}^{N}\left(z_{h}^{n-1}(u)-z^{n}, u^{n}-u_{h}^{n}\right) \\
\quad+k \sum_{n=1}^{N}\left(\gamma u_{h}^{n}+z_{h}^{n-1}, u_{h}^{n}-u^{n}\right) \\
\leq 0
\end{aligned}
$$




$$
\begin{gathered}
=k \sum_{n=1}^{N}\left(z_{h}^{n-1}\left(u^{n}\right)-z^{n-1}, u^{n}-u_{h}^{n}\right) \\
+k \sum_{n=1}^{N}\left(z^{n-1}-z^{n}, u^{n}-u_{h}^{n}\right) .
\end{gathered}
$$

Using Young inequality we obtain

$$
\|\| u-u_{h}\left\|\left.\right|_{L^{2}\left(0, T, L^{2}(\Omega)\right)} \leq C h^{1 / 2}+C k\right\| \frac{\partial z}{\partial t} \|_{L^{2}\left(0, T, L^{2}(\Omega)\right)}
$$

Theorem 14. Let $(y, \mathbf{q}, z, \mathbf{p}, u)$ and $\left(y_{h}^{n}, \mathbf{q}_{h}^{n}, z_{h}^{n-1}, \mathbf{p}_{h}^{n-1}, u_{h}^{n}\right)$ be the solutions of (11) and (17), respectively. Then we have

$$
\begin{aligned}
\mid \| u- & u_{h}\left\|\left.\right|_{L^{2}\left(0, T, L^{2}(\Omega)\right)}+\left|\left\|\left(y-y_{h}, \mathbf{q}-\mathbf{q}_{h}\right)\right\|\right|_{\diamond}\right. \\
+ & \left|\left\|\left(z-z_{h}, \mathbf{p}-\mathbf{p}_{h}\right)\right\|\right|_{\diamond} \leq C\left(h^{1 / 2}+k\right) .
\end{aligned}
$$

Proof. By Lemma 9 and using triangle inequality we derive

$$
\begin{aligned}
& \left|\left\|\left(y-y_{h}, \mathbf{q}-\mathbf{q}_{h}\right)\right\|_{\diamond}^{2} \leq\right|\left\|\left(y-y_{h}(u), \mathbf{q}-\mathbf{q}_{h}(u)\right)\right\|_{\diamond}^{2} \\
& +\left|\left\|\left(y_{h}(u)-y_{h}, \mathbf{q}_{h}(u)-\mathbf{q}_{h}\right)\right\|\right|_{\diamond}^{2} \\
& \quad \leq C h\left(\|\mathbf{q}\|_{L^{\infty}\left(0, T ; H^{1 / 2}(\Omega)\right)}^{2}+\|y\|_{L^{\infty}\left(0, T ; H^{1}(\Omega)\right)}^{2}\right. \\
& \left.+\left\|\mathbf{q}_{t}\right\|_{L^{2}\left(0, T ; H^{1 / 2}(\Omega)\right)}^{2}+\left\|y_{t}\right\|_{L^{2}\left(0, T ; H^{1}(\Omega)\right)}^{2}\right) \\
& +C k^{2}\left\|\frac{\partial^{2} y}{\partial t^{2}}\right\|_{L^{2}\left(0, T ; L^{2}(\Omega)\right)}^{2}+C\left|\left\|u-u_{h}\right\|\right|_{L^{2}\left(0, T ; L^{2}(\Omega)\right)}^{2}
\end{aligned}
$$

By Lemma 10 we deduce that

$$
\begin{aligned}
& \|\|\left(z-z_{h}, \mathbf{p}-\mathbf{p}_{h}\right)\|\|_{\diamond}^{2} \leq \mid\left\|\left(z-z_{h}(u), \mathbf{p}-\mathbf{p}_{h}(u)\right)\right\|_{\diamond}^{2} \\
& \quad+\|\|\left(z_{h}(u)-z_{h}, \mathbf{p}_{h}(u)-\mathbf{p}_{h}\right) \|_{\diamond}^{2} \\
& \quad \leq C h\left(\|\mathbf{p}\|_{L^{\infty}\left(0, T ; H^{1 / 2}(\Omega)\right)}^{2}+\|z\|_{L^{\infty}\left(0, T ; H^{1}(\Omega)\right)}^{2}\right. \\
& \quad+\left\|\mathbf{p}_{t}\right\|_{L^{2}\left(0, T ; H^{1 / 2}(\Omega)\right)}^{2}+\left\|z_{t}\right\|_{L^{2}\left(0, T ; H^{1}(\Omega)\right)}^{2} \\
& \left.+\|y\|_{L^{\infty}\left(0, T ; H^{1}(\Omega)\right)}^{2}\right)+C \mid\left\|u-u_{h}\right\|_{L^{2}\left(0, T ; L^{2}(\Omega)\right)}^{2} \\
& \quad+C k^{2}\left(\left\|y_{t}\right\|_{L^{2}\left(0, T ; L^{2}(\Omega)\right)}^{2}+\left\|\frac{\partial y_{d}}{\partial t}\right\|_{L^{2}\left(0, T ; L^{2}(\Omega)\right)}^{2}\right. \\
& \left.\quad+\left\|\frac{\partial^{2} z}{\partial t^{2}}\right\|_{L^{2}\left(0, T ; L^{2}(\Omega)\right)}^{2}\right) .
\end{aligned}
$$

Combining Lemma 13, (135), and (136) we arrive at

$$
\begin{aligned}
\mid \| u & -u_{h}\left\|_{L^{2}\left(0, T, L^{2}(\Omega)\right)}+\mid\right\|\left(y-y_{h}, \mathbf{q}-\mathbf{q}_{h}\right) \|_{\diamond} \\
& +\left.\left\|\left(z-z_{h}, \mathbf{p}-\mathbf{p}_{h}\right)\right\|\right|_{\diamond} \leq C h^{1 / 2}\left(\|\mathbf{q}\|_{L^{\infty}\left(0, T ; H^{1 / 2}(\Omega)\right)}\right. \\
& +\|y\|_{L^{\infty}\left(0, T ; H^{1}(\Omega)\right)}+\left\|\mathbf{q}_{t}\right\|_{L^{2}\left(0, T ; H^{1 / 2}(\Omega)\right)} \\
& \left.+\left\|y_{t}\right\|_{L^{2}\left(0, T ; H^{1}(\Omega)\right)}\right)+C h^{1 / 2}\left(\|\mathbf{p}\|_{L^{\infty}\left(0, T ; H^{1 / 2}(\Omega)\right)}\right. \\
& +\|z\|_{L^{\infty}\left(0, T ; H^{1}(\Omega)\right)}+\left\|\mathbf{p}_{t}\right\|_{L^{2}\left(0, T ; H^{1 / 2}(\Omega)\right)} \\
& \left.+\left\|z_{t}\right\|_{L^{2}\left(0, T ; H^{1}(\Omega)\right)}\right)+C k\left(\left\|y_{t}\right\|_{L^{2}\left(0, T ; L^{2}(\Omega)\right)}\right. \\
& +\left\|\frac{\partial y_{d}}{\partial t}\right\|_{L^{2}\left(0, T ; L^{2}(\Omega)\right)}+\left\|\frac{\partial^{2} y}{\partial t^{2}}\right\|_{L^{2}\left(0, T ; L^{2}(\Omega)\right)} \\
& \left.+\left\|\frac{\partial^{2} z}{\partial t^{2}}\right\|_{L^{2}\left(0, T ; L^{2}(\Omega)\right)}\right),
\end{aligned}
$$

which completes the theorem.

\section{Competing Interests}

The authors declare that there is no conflict of interests regarding the publication of this paper.

\section{Acknowledgments}

The authors would like to acknowledge the support of Natural Science Foundation of Shandong Province (no. ZR2016JL004).

\section{References}

[1] J. Zhu and Q. C. Zeng, "A mathematical theoretical frame for control of air pollution," Science China Earth Sciences D, vol. 32, pp. 864-870, 2002.

[2] A. Martínez, C. Rodríguez, and M. E. Vazquez-Mendez, “Theoretical and numerical analysis of an optimal control problem related to wastewater treatment," SIAM Journal on Control and Optimization, vol. 38, no. 5, pp. 1534-1553, 2000.

[3] R. Becker and B. Vexler, "Optimal control of the convectiondiffusion equation using stabilized finite element methods," Numerische Mathematik, vol. 106, no. 3, pp. 349-367, 2007.

[4] M. Braack, "Optimal control in fluid mechanics by finite elements with symmetric stabilization," SIAM Journal on Control and Optimization, vol. 48, no. 2, pp. 672-687, 2009.

[5] S. S. Collis and M. Heinkenschloss, "Analysis of the streamline upwind/Petrov Galerkin method applied to the solution of optimal control problems," Tech. Rep. TR02-01, Department of Computational and Applied Mathematics, Rice University, Houston, Tex, USA, 2002.

[6] M. Hinze, N. Yan, and Z. Zhou, "Variational discretization for optimal control governed by convection dominated diffusion equations," Journal of Computational Mathematics, vol. 27, no. 2-3, pp. 237-253, 2009. 
[7] T. Sun, "Discontinuous Galerkin finite element method with interior penalties for convection diffusion optimal control problem," International Journal of Numerical Analysis and Modeling, vol. 7, no. 1, pp. 87-107, 2010.

[8] N. Yan and Z. Zhou, "A RT mixed FEM/DG scheme for optimal control governed by convection diffusion equations," Journal of Scientific Computing, vol. 41, pp. 273-299, 2009.

[9] H. Yücel, M. Heinkenschloss, and B. Karasözen, "An adaptive discontinuous Galerkin method for convection dominated distributed optimal control problems," Tech. Rep., Department of Computational and Applied Mathematics, Rice University, Houston, Tex, USA, 2012.

[10] H. Yücel, M. Heinkenschloss, and B. Karasözen, "Distributed optimal control of diffusion-convection-reaction equations using discontinuous Galerkin methods," in Numerical Mathematics and Advanced Applications, vol. 2011, pp. 389-397, Springer, Heidelberg, Germany, 2013.

[11] Z. Zhou and N. Yan, "The local discontinuous Galerkin method for optimal control problem governed by convection diffusion equations," International Journal of Numerical Analysis and Modeling, vol. 7, no. 4, pp. 681-699, 2010.

[12] Z. Zhou, "A posteriori error estimates for discontinuous Galerkin approximation of non-stationary convection-diffusion optimal control problems," International Journal of Computer Mathematics, vol. 93, no. 12, pp. 2106-2123, 2016.

[13] H. Fu, "A characteristic finite element method for optimal control problems governed by convection-diffusion equations," Journal of Computational and Applied Mathematics, vol. 235, no. 3, pp. 825-836, 2010.

[14] Z. J. Zhou and N. N. Yan, "A survey of numerical methods for convection-diffusion optimal control problems," Journal of Numerical Mathematics, vol. 22, no. 1, pp. 61-85, 2014.

[15] J. Wang and N. Yan, "A parallel domain decomposition procedure for convection diffusion problems," in Domain Decomposition Methods in Sciences and Engineering (Beijing, 1995), pp. 333-339, Wiley, Chichester, UK, 1997.

[16] P. A. Raviart and J. M. Thomas, "A mixed finite element method for 2nd order elliptic problems," in Mathematical Aspects of Finite Element Methods, vol. 606 of Lecture Notes in Mathematics, pp. 292-315, Springer, Berlin, Germany, 1977.

[17] F. Brezzi and M. Fortin, Mixed and Hybrid Finite Element Methods, vol. 15 of Springer Series in Computational Mathematics, Springer-Verlag, Berlin, Germany, 1991.

[18] C. Johnson and J. Pitkränta, "An analysis of the discontinuous Galerkin method for a scalar hyperbolic equation," Mathematics of Computation, vol. 46, no. 173, pp. 1-26, 1986. 


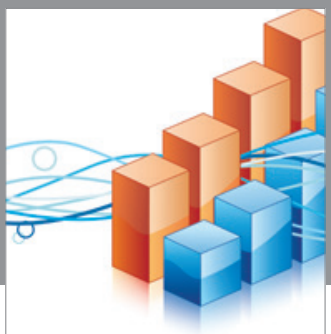

Advances in

Operations Research

vatem alat4

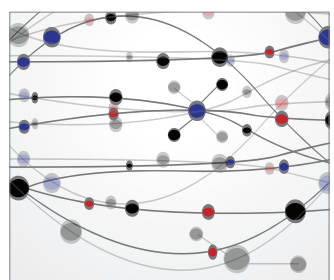

\section{The Scientific} World Journal
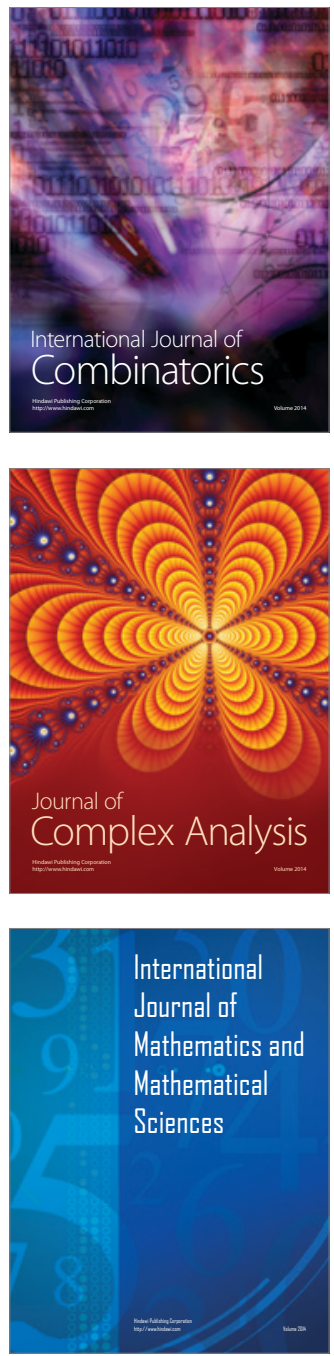
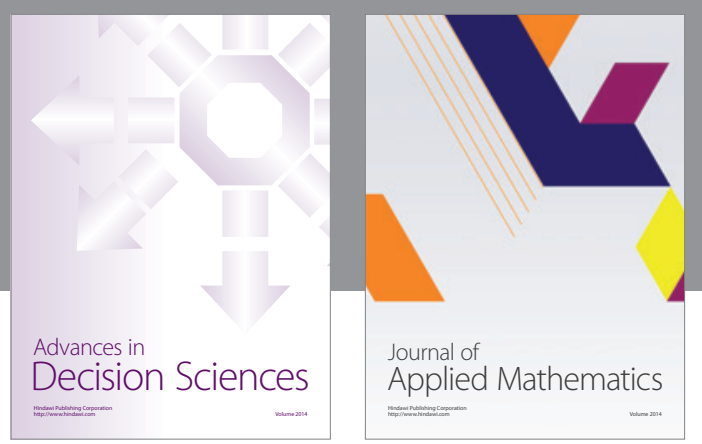

Algebra

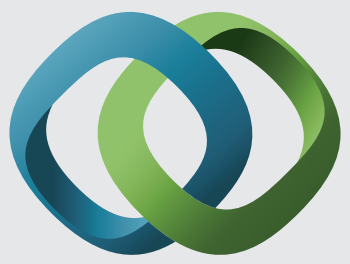

\section{Hindawi}

Submit your manuscripts at

https://www.hindawi.com
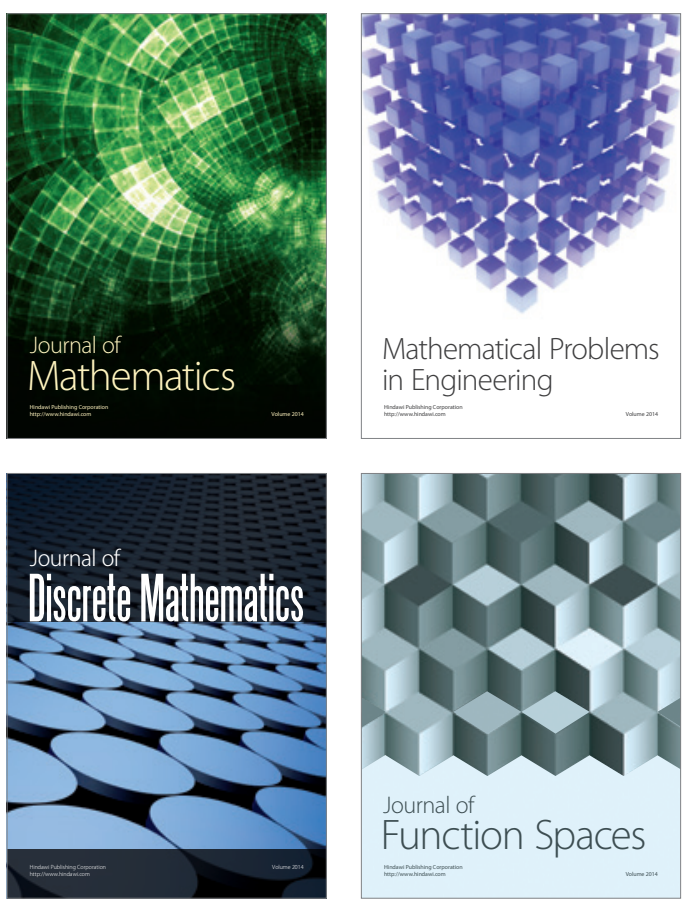

Mathematical Problems in Engineering
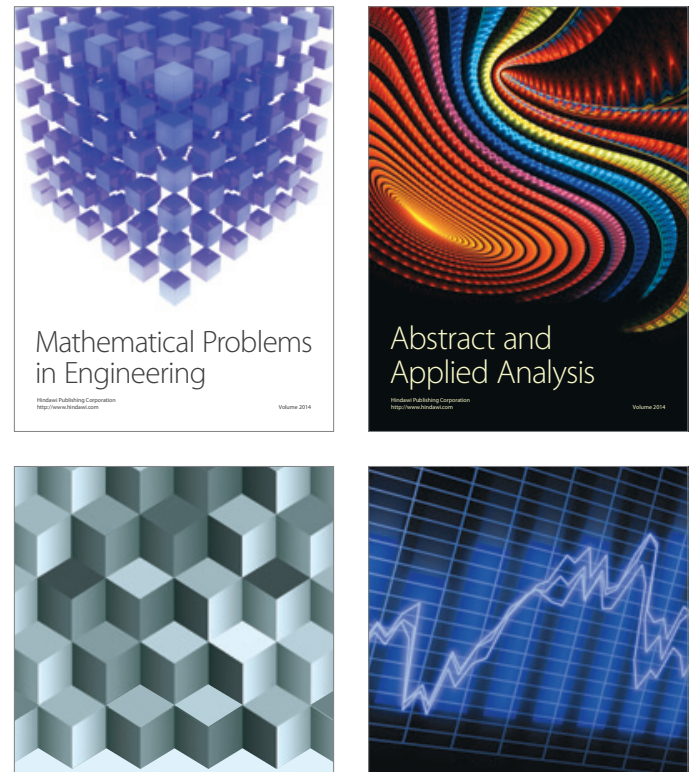

Journal of

Function Spaces

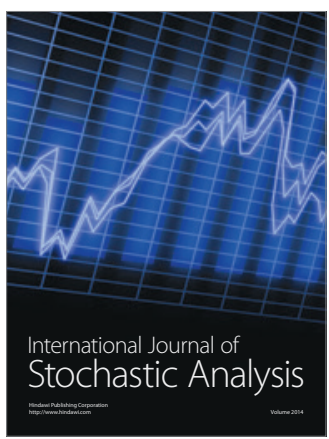

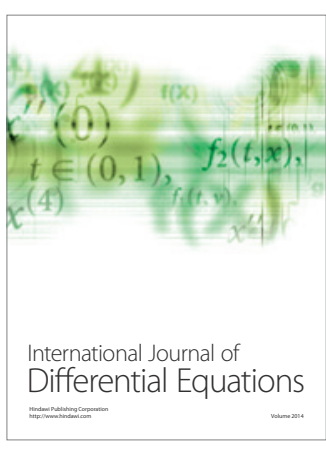
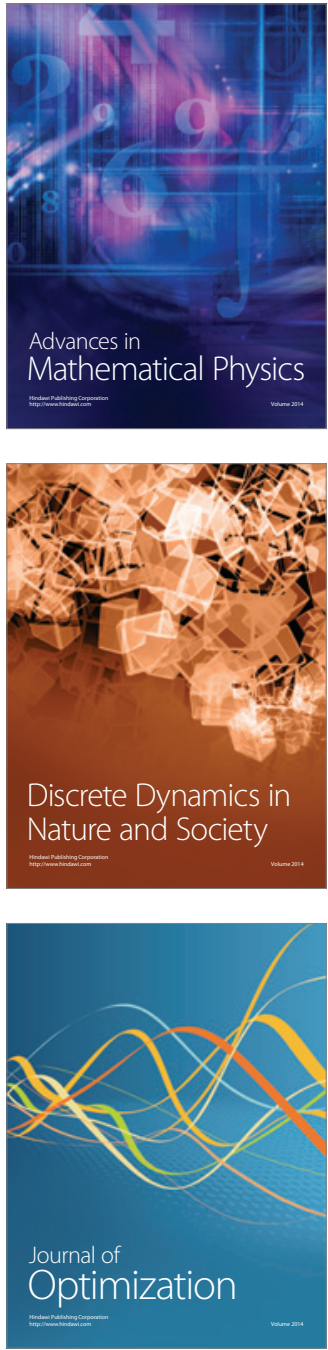